\title{
From topological strings to minimal models
}

\author{
Omar Foda ${ }^{a}$ and Jian-Feng $\mathbf{W} \mathbf{u}^{b, c}$ \\ ${ }^{a}$ School of Mathematics and Statistics, University of Melbourne, \\ Royal Parade, Parkville, VIC 3010, Australia \\ ${ }^{b}$ Department of Mathematics and Statistics, Henan University, \\ Minglun Street, Kaifeng city, Henan, China \\ ${ }^{c}$ Beijing Institute of Theoretical Physics and Mathematics, \\ 3rd Shangdi Street, Beijing, China \\ E-mail: omar.foda@unimelb.edu.au, muchen.wu@gmail.com
}

ABstract: We glue four refined topological vertices to obtain the building block of 5D $\mathrm{U}(2)$ quiver instanton partition functions. We take the $4 \mathrm{D}$ limit of the result to obtain the building block of 4D instanton partition functions which, using the AGT correspondence, are identified with Virasoro conformal blocks.

We show that there is a choice of the parameters of the topological vertices that we start with, as well as the parameters and the intermediate states involved in the gluing procedure, such that we obtain Virasoro minimal model conformal blocks.

Keywords: Duality in Gauge Field Theories, Conformal and W Symmetry, Topological Strings, Integrable Field Theories

ArXiv EPRINT: 1504.01925 


\section{Contents}

1 Introduction $\quad 2$

1.1 Background and basic concepts 2

1.1.1 Topological strings 2

1.1.2 From topological strings to 5D gauge theories 2

1.1.3 Refined partition functions 2

1.1.4 The refined topological vertex 2

1.1.5 From 5D to 4D gauge theories 3

1.1.6 From 4D gauge theories to generic 2D conformal field theories 3

1.1.7 From generic conformal field theories to minimal models 3

1.2 Plan of this work 3

1.3 Outline of contents 4

2 The refined topological vertex 5

2.1 Partitions and Young diagrams 5

2.1.1 Partitions 5

2.1.2 Young diagrams 5

2.1.3 Cells 5

2.1.4 Arms and legs, half-extended and extended 5

2.1.5 Remark 5

2.1.6 Partition pairs 6

2.1.7 Sum of row-lengths and squares of row-lengths 6

$\begin{array}{lll}2.1 .8 & \text { Sequences } & 6\end{array}$

2.1.9 A function of the arm-lengths and the leg-lengths 7

$\begin{array}{ll}2.1 .10 \text { Remark } & 7\end{array}$

$\begin{array}{lll}2.2 & \text { Schur and skew-Schur functions } & 7\end{array}$

2.3 Topological vertices $\quad 7$

2.3.1 The topological vertex of Aganagic et al. $\quad 7$

$\begin{array}{lll}2.3 .2 & \text { The refined topological vertex } & 8\end{array}$

$\begin{array}{lll}\text { 2.3.3 } & \text { On framing vectors and framing factors } & 8\end{array}$

3 A 5D U(2) basic web partition function $\quad 8$

3.1 Geometric engineering 8

$\begin{array}{lll}\text { 3.1.1 Two skew Schur function identities } & 10\end{array}$

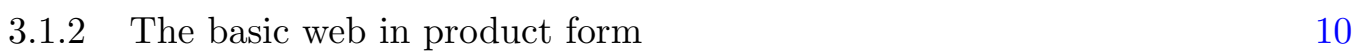

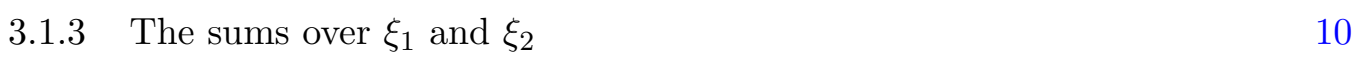

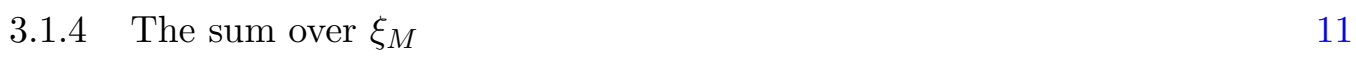

3.1.5 The sums over $\eta_{1}$ and $\eta_{2} \quad 11$

$\begin{array}{lll}3.1 .6 & \text { The sum over } \tau & 11\end{array}$

$\begin{array}{lll}3.1 .7 & \text { Normalised products } & 12\end{array}$

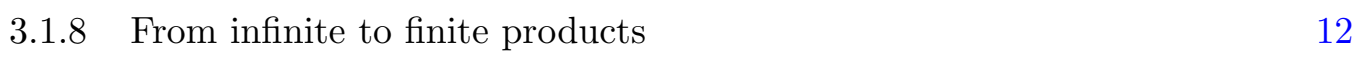


3.1.10 Remark 13

4 A 4D U(2) basic web partition function $\quad 13$

$\begin{array}{lll}4.1 \text { Two parameters } & 13\end{array}$

5 The building block of the $4 \mathrm{D} \mathrm{U}(2)$ quiver instanton partition function 14

$6 \quad$ Identification of $\mathcal{W}_{\mathrm{VW} \Delta}^{\text {norm }}$ and $\mathcal{Z}_{\text {building.block }}^{4 \mathrm{D}}$. The numerators $\quad 15$

7 Identification of $\mathcal{W}_{\mathrm{VW} \Delta}^{\text {norm }}$ and $\mathcal{Z}_{\text {building.block }}^{4 \mathrm{D}}$. The denominators 15

$\begin{array}{lll}7.1 & \text { Linear conformal blocks } & 17\end{array}$

$\begin{array}{ll}7.2 \text { Cyclic conformal blocks } & 17\end{array}$

$\begin{array}{lll}7.3 & \text { The denominator } z_{\text {den }}^{\prime} \text { and the Burge conditions } & 17\end{array}$

8 Restricted instanton partition functions for $\mathcal{M}^{p, p^{\prime}, \mathcal{H}}$. The parameters 17

$\begin{array}{ll}\text { 8.1 AGT parameterisation of minimal models } & 17\end{array}$

$\begin{array}{ll}8.2 \text { Two sets of charges in minimal models } & 18\end{array}$

9 From gauge theory parameters to minimal model parameters $\quad 18$

$\begin{array}{lll}9.1 \text { The fusion rules } & 18\end{array}$

10 Restricted instanton partition functions for $\mathcal{M}^{p, p^{\prime}, \mathcal{H}}$. The partition $\begin{array}{lr}\text { pairs } & 18\end{array}$

$\begin{array}{ll}10.1 \text { The initial four products } & 19\end{array}$

$\begin{array}{ll}10.2 \text { Two zero-conditions } & 19\end{array}$

10.3 From two zero-conditions to one zero-condition 19

$\begin{array}{ll}10.4 \text { One non-zero condition } & 20\end{array}$

$\begin{array}{ll}\text { 10.4.1 Remark } & 20\end{array}$

$\begin{array}{ll}10.5 \text { The first product } & 20\end{array}$

10.5.1 From two zero-conditions to one non-zero-condition 20

$\begin{array}{ll}10.5 .2 \text { The stronger condition } & 21\end{array}$

$\begin{array}{ll}10.6 \text { The second product } & 21\end{array}$

10.6.1 From two zero-conditions to one non-zero-condition 21

$\begin{array}{ll}\text { 10.6.2 The stronger condition } & 21\end{array}$

$\begin{array}{ll}10.7 \text { The third product } & 21\end{array}$

$\begin{array}{ll}\text { 10.7.1 The stronger condition } & 22\end{array}$

$\begin{array}{ll}10.8 \text { The fourth product } & 22\end{array}$

10.8.1 The stronger condition $\quad 22$

$\begin{array}{ll}10.9 \text { The Burge conditions } & 22\end{array}$

11 Comments and open questions $\quad 23$ 


\section{Introduction}

We plan to start from the refined topological vertex and obtain Virasoro A-series minimal model conformal blocks times Heisenberg factors.

\subsection{Background and basic concepts}

\subsubsection{Topological strings}

Perturbative string theory, seen from a world-sheet point of view, is a $2 \mathrm{D}$ conformal field theory, coupled to 2D gravity, on Riemann surfaces. If the 2D conformal field theory is topological, in the sense that the correlation functions are independent of the metrics on the Riemann surfaces, the resulting string theory is topological. For an introduction, we refer to [1], and references therein. There are two constructions of topological string theories, the A-model and the B-model. For the purposes of this note, it suffices to say that we work are in the context of the A-model, and that the target space, that the strings propagate in, is $\mathbb{R}^{1,3} \times \mathcal{X}$, where $\mathcal{X}$ is a toric Calabi-Yau complex 3-manifold. For suitable choices of $\mathcal{X}$, topological strings are non-trivial but tractable, and one can compute their partition functions. For further details, we refer to the reviews [2, 3], and references therein.

\subsubsection{From topological strings to 5D gauge theories}

Topological string partition functions are interesting in themselves, and for yet another, an a priori unexpected reason. Namely, for suitable choices of the Calabi-Yau manifold $\mathcal{X}$, one can compute the topological string partition function $\mathcal{Z}^{\text {top }}$, and moreover, identify the result with the instanton partition function $\mathcal{Z}_{\text {instanton }}^{5 \mathrm{D}}$ of a corresponding $5 \mathrm{D} \mathcal{N}=2$ supersymmetric quiver gauge theory, thereby 'geometrically-engineering' the latter theory $[4,5]$.

\subsubsection{Refined partition functions}

A $5 \mathrm{D}$ instanton partition function depends, in general, on two deformation parameters, $\epsilon_{1}$ and $\epsilon_{2}$. For $\epsilon_{1}+\epsilon_{2}=0$, the instanton partition function is unrefined. For $\epsilon_{1}+\epsilon_{2} \neq 0$, the instanton partition function is refined. Given the identification of 5D instanton partition function and topological strings, the latter also depend, in general on the deformation parameters, $\epsilon_{1}$ and $\epsilon_{2}$. For $\epsilon_{1}+\epsilon_{2}=0$, the topological string partition function is unrefined. For $\epsilon_{1}+\epsilon_{2} \neq 0$, the instanton partition function is refined.

\subsubsection{The refined topological vertex}

To compute topological string partition functions, one splits the full partition function into basic building blocks, computes the contribution of each building block, then combines the contributions to obtain the required result. In the unrefined case, $\epsilon_{1}+\epsilon_{2}=0$, this was achieved in [6]. In this case, the building blocks are copies of the original, unrefined 'topological vertex' introduced in [6]. In the refined case, $\epsilon_{1}+\epsilon_{2} \neq 0$, this was achieved in [7-9] In this case, the building blocks are copies of the 'refined topological vertex' [7-9]. 


\subsubsection{From 5D to 4D gauge theories}

A $5 \mathrm{D}$ quiver gauge theory instanton partition function, $\mathcal{Z}_{\text {instanton }}^{5 \mathrm{D}}$, depends on the radius $R$ of a space-like circle. In the limit $R \rightarrow 0, \mathcal{Z}_{\text {instanton }}^{5 \mathrm{D}}$ reduces to a corresponding $4 \mathrm{D}$ instanton partition function $\mathcal{Z}_{\text {instanton }}^{4 \mathrm{D}}[9-14]$. The deformation parameters $\epsilon_{1}$ and $\epsilon_{2}$ of the $5 \mathrm{D}$ theory are inherited by the $4 \mathrm{D}$ theory.

\subsubsection{From 4D gauge theories to generic 2D conformal field theories}

The $4 \mathrm{D}$ instanton partition functions $\mathcal{Z}_{\text {instanton }}^{4 \mathrm{D}}$ are identified via the AGT correspondence with 2D Virasoro generic conformal blocks times Heisenberg factors, $\mathcal{B}^{\text {gen, }} \mathcal{H}$ [15]. In its original formulation, the AGT correspondence applies only to generic, that is non-minimal conformal field theories. From 4D instanton partition functions with $\epsilon_{1}+\epsilon_{2}=0$, one obtains conformal blocks of the conformal field theory of a Gaussian free field at the free fermion point. From $4 \mathrm{D}$ instanton partition functions with $\epsilon_{1}+\epsilon_{2} \neq 0$, one obtains conformal blocks of non-Gaussian, but also non-minimal conformal field theories.

\subsubsection{From generic conformal field theories to minimal models}

One can choose the parameters that appear in $\mathcal{Z}_{\text {instanton, }}^{4 \mathrm{D}}$ and restrict the states that are allowed as intermediate states, in such a way that one obtains restricted $4 \mathrm{D}$ instanton partition functions $\mathcal{Z}_{\text {instanton }}^{4 \mathrm{D} \text { min }}$ that can be identified with conformal blocks in Virasoro $A$ series minimal models times Heisenberg factors $[16,17]$. In particular, as we will show in the sequel, choosing $\epsilon_{1}=-\sqrt{p / p^{\prime}}$, and $\epsilon_{2}=\sqrt{p^{\prime} / p}$, where $p$ and $p^{\prime}$ are two co-prime positive integers, $0<p<p^{\prime}$, one obtains conformal blocks in a minimal model parameterised by $p$ and $p^{\prime}$.

\subsection{Plan of this work}

From the above chain of connections, it is expected that one can start from $\mathcal{Z}^{\text {reftop }}$, choose the parameters and restrict the intermediate states to obtain 5D instanton partition functions $\mathcal{Z}_{\text {instanton }}^{5 \mathrm{D} \text {, min }}$, reduce to the corresponding $\mathcal{Z}_{\text {instanton }}^{4 \mathrm{D}, \text { min }}$, and compute minimal model conformal blocks from the latter. In this note, we work out the above chain of connections, which amounts to extending the result of $[16,17]$ by starting from topological strings and topological vertices rather than from $4 \mathrm{D}$ instanton partition functions. We glue four refined topological vertices to obtain the building block of the 5D instanton partition functions, and take the $4 \mathrm{D}$ limit of the latter to obtain the building block of the $4 \mathrm{D}$ instanton partition functions $\mathcal{Z}_{\text {building.block }}^{4 \mathrm{D}}$. From that point on, we use the results of $[16,17]$ to obtain conformal blocks in minimal models.

Since $\mathcal{Z}_{\text {building.block }}^{4 \mathrm{D}}$, which can be regarded as the starting point of the results in [16, 17], is constructed here from refined topological vertices, the construction in this note is, in this sense, more basic than that in $[16,17]$.

The Virasoro $A$-series minimal conformal blocks that we obtain can be computed using other methods, but we view this note as an accessible introduction to one more approach to the minimal conformal blocks that, hopefully, can be extended to minimal blocks beyond 
what can currently be computed, including the $W_{N}$ minimal blocks that do not satisfy the conditions of $[18,19] .^{1}$

\subsection{Outline of contents}

To simplify the presentation, we divide the content into short sections. In 2, we recall basic definitions related to Young diagrams and Schur functions, followed by the definition of the refined topological vertex $\mathcal{C}_{\lambda \mu \nu}[q, t]$ of Iqbal, Kozcaz and Vafa [9], where $\lambda, \mu$, and $\nu$ are Young diagrams, $q$ and $t$ are parameters. In 3, we glue four copies of $\mathcal{C}_{\lambda \mu \nu}[q, t]$ to build a $\mathrm{U}(2)$ basic web partition function $\mathcal{W}_{\mathbf{V} \mathbf{W} \boldsymbol{\Delta}}[q, t, R]$, where each of $\mathbf{V}$ and $\mathbf{W}$ is a pair of Young diagrams, and $\boldsymbol{\Delta}=\left\{\Delta_{1}, \Delta_{M}, \Delta_{2}\right\}$ are Kähler parameters. In 4 , we set $q=e^{R \epsilon_{2}}, t=$ $e^{-R \epsilon_{1}}$, where $\epsilon_{1}$ and $\epsilon_{2}$ are Nekrasov's regularisation parameters, then take the limit $R \rightarrow 0$, to obtain $\mathcal{W}_{\mathbf{V} \mathbf{W} \boldsymbol{\Delta}}\left[\epsilon_{1}, \epsilon_{2}, R \rightarrow 0\right]$. In 5 , we recall the definition of the normalised contribution of the bifundamental hypermultiplet, $\mathcal{Z}_{\text {building.block }}^{4 \mathrm{D}}$ to $\mathcal{Z}_{\text {instanton }}^{4 \mathrm{D}}$. In 6 , we compare the numerators of $\mathcal{W}_{\mathbf{V}, \mathbf{W}, \boldsymbol{\Delta}}^{\text {norm }}$ and $\mathcal{Z}_{\text {building.block }}^{4 \mathrm{D}}$, and identify the Kähler parameters with gauge theory parameters. In 7 , we compare the denominators of $\mathcal{W}_{\mathbf{V}, \mathbf{W}, \boldsymbol{\Delta}}^{\text {norm }}$ and $\mathcal{Z}_{\text {building.block }}^{4 \mathrm{D}}$, and show that there is a normalisation such that the two denominators agree, without changing the results of the computations of the instanton partition functions. In 8 , we recall the choice of parameters that allows us to use $\mathcal{Z}_{\text {building.block }}^{4 \mathrm{D}}$ to build $4 \mathrm{D}$ instanton partition functions that can be identified with Virasoro $A$-series minimal model conformal blocks times Heisenberg factors. In 9, we identify the parameters of $\mathcal{W}_{\mathbf{V W} \boldsymbol{\Delta}}^{\text {norm }}$ and of $\mathcal{Z}_{\text {building.block }}^{4 \mathrm{D}}$. In 10, we outline a proof, following [17], of the statement that we need to impose Burge conditions on the partition pairs that appear in our constructions, to make the topological string partition functions free of non-physical singularities, once we choose the parameters to coincide with those of the minimal model. Section 11 includes comments and remarks.

\section{Abbreviations and notation}

We focus on $\mathrm{U}(2)$ quiver gauge theories, and simply say 'the instanton partition functions'. We assume that every conformal block, whether Liouville or minimal, includes a factor from a field theory of a free boson on a line, and omit 'times Heisenberg factors'. The normalised contribution of the bifundamental hypermultiplet is understood as the building block of the instanton partition function, as all other contributions can be obtained from it. We simply say 'the bifundamental partition function'.

Topological string-related notation. $\mathcal{C}_{\lambda \mu \nu}[q, t]$ is the refined topological vertex. $\mathcal{W}_{\mathbf{V} \mathbf{W} \boldsymbol{\Delta}}[q, t, R]$ is a basic web refined topological string partition function. $\mathbf{V}$ and $\mathbf{W}$ are two pairs of Young diagrams. $\boldsymbol{\Delta}$ is a set of three Kähler parameters. $q$ and $t$ are deformation parameters. $R$ is the radius of a space-like circle. ${ }^{2} \mathcal{Z}^{\text {ref top }}$ is the topological string partition function.

\footnotetext{
${ }^{1}$ Expressions for the 3-point functions of $W_{N}$ Toda theory were proposed, starting from topological strings, in [20], and checked in [21]. These results should be useful in subsequent studies of $W_{N}$ minimal models.

${ }^{2}$ The $5 \mathrm{D} \mathrm{U}(2)$ quiver theories in this note live on D5-branes in time-like $x^{0}$, and space-like $x^{1}, x^{2}, x^{3}, x^{5}$ and $x^{6}$ dimensions. Following [22], we take $x^{5}$ to be a circle of radius $R, \beta$ in [22], such that setting $R \rightarrow 0$ is equivalent to taking the $4 \mathrm{D}$ limit. For a complete discussion, we refer to [22].
} 
Gauge theory-related notation. $\quad \mathcal{Z}_{\text {instanton }}^{5 \mathrm{D}}$ is the $5 \mathrm{D} \mathrm{U}(2)$ quiver gauge theory instanton partition functions. $\mathcal{Z}_{\text {instanton }}^{4 \mathrm{D}}$ is the $4 \mathrm{D} \mathrm{U}(2)$ quiver gauge theory instanton partition functions. $\mathcal{Z}_{\text {building.block }}^{4 \mathrm{D}}$ is the normalised contribution of the bifundamental hypermultiplet. The parameters $\epsilon_{1}$ and $\epsilon_{2}$ are deformation parameters.

Conformal field theory-related notation. $\mathcal{B}^{\text {gen, } \mathcal{H}}$ are $2 \mathrm{D}$ Virasoro generic conformal blocks times Heisenberg factors. $\mathcal{B}^{\text {gen, min }}$ are $2 \mathrm{D}$ Virasoro A-series minimal model conformal blocks times Heisenberg factors. The parameters $p$ and $p^{\prime}$ are coprime positive integers that label a Virasoro minimal model. The parameters $r$ and $s$ are integers that satisfy $0<r<p, 0<s<p^{\prime}$ and label Virasoro minimal model highest weight representations.

\section{The refined topological vertex}

We recall basic definitions related to Young diagrams and Schur functions, followed by the definition of the refined topological vertex.

\subsection{Partitions and Young diagrams}

\subsubsection{Partitions}

A partition $\pi$ of a non-negative integer $|\pi|$ is a set of non-negative integers $\left\{\pi_{1}, \pi_{2}, \cdots, \pi_{p}\right\}$, where $p$ is the number of parts, $\pi_{i} \geqslant \pi_{i+1}$, and $\sum_{i=1}^{p} \pi_{i}=|\pi|$.

\subsubsection{Young diagrams}

A partition $\pi$ is represented as a Young diagram $Y$, as in figure 1, which is a set of $p$ rows $\left\{y_{1}, y_{2}, \cdots, y_{p}\right\}$, such that row- $i$ has $y_{i}=\pi_{i}$ cells, ${ }^{3} y_{i} \geqslant y_{i+1}$, and $\sum_{i} y_{i}=|Y|=|\pi|$. We use $Y^{\top}$ for the transpose of $Y$.

\subsubsection{Cells}

We use $\square$ for a cell, or a square in the south-east quadrant of the plane, and refer to the coordinates of $\square$ as $\{\mathrm{R}, \mathrm{c}\}$. If $\square$ is inside a Young diagram $Y$, then $\mathrm{R}$ is the $Y$-row-number, counted from top to bottom, and $\mathrm{c}$ is the $Y$-column-number, counted from left to right, that $\square$ lies in. If $\square$ is outside $Y$, we still regard $\mathrm{R}$ as a $Y$-row-number, albeit the length of this row is zero, and we still regard c as a $Y$-column-number, albeit the length of this column is zero.

\subsubsection{Arms and legs, half-extended and extended}

Consider a cell $\square$ that has coordinates $\{\mathrm{R}, \mathrm{c}\}$. We define the lengths of the arm $A_{\square, Y}$, halfextended arm $A_{\square, Y}^{+}$, extended arm $A_{\square, Y}^{++}$, the leg $L_{\square, Y}$, half-extended leg $L_{\square, Y}^{+}$, extended $\operatorname{leg} L_{\square, Y}^{++}$, of $\square$ with respect to the Young diagram $Y$, to be

$$
\begin{array}{lll}
A_{\square, Y}=y_{\mathrm{R}}-\mathrm{c}, & A_{\square, Y}^{+}=A_{\square, Y}+\frac{1}{2}, & A_{\square, Y}^{++}=A_{\square, Y}+1, \\
L_{\square, Y}=y_{\mathrm{C}}^{\top}-\mathrm{R}, & L_{\square, Y}^{+}=L_{\square, Y}+\frac{1}{2}, & L_{\square, Y}^{++}=L_{\square, Y}+1,
\end{array}
$$

\subsubsection{Remark}

$A_{\square, Y}$ and $L_{\square, Y}$ can be negative when $\square$ lies outside $Y$.

\footnotetext{
${ }^{3}$ We use $y_{i}$ for $i$-th row as well as for the number of cells in that row.
} 


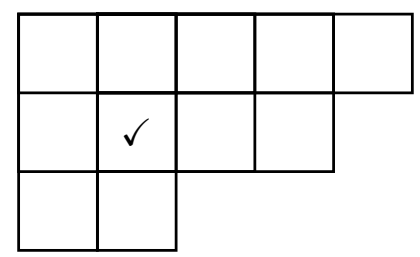

Figure 1. The Young diagram $Y$ of $5+4+2$. The rows are numbered from top to bottom. The columns are numbered from left to right. The checkmarked cell has $A=2, A^{+}=\frac{5}{2}, A^{++}=3$, $L=1$, etc.
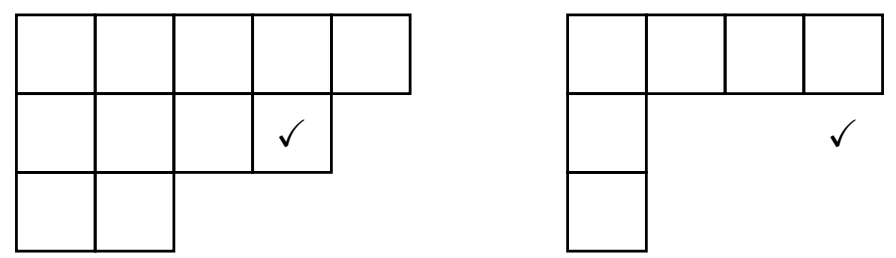

Figure 2. A partition pair $\{\mu, \nu\} . \mu$ is on the left, $\nu$ is on the right. The checkmarked cell at coordinates $(2,4)$, in the lower right quadrant of the plane, is in $\mu$, but not in $\nu$, and has $A_{\mu}=0$, $A_{\mu}^{+}=\frac{1}{2}, A_{\mu}^{++}=1, L_{\mu}=0, L_{\mu}^{+}=\frac{1}{2}, L_{\mu}^{++}=1$, as well as $A_{\nu}=-3$, etc. and $L_{\nu}=-1$, etc.

\subsubsection{Partition pairs}

A partition pair $\mathbf{Y}$ is a set of two Young diagrams, $\left\{Y^{1}, Y^{2}\right\}$, as in figure 2 , where $|\mathbf{Y}|=$ $\left|\mathbf{Y}^{\mathbf{1}}\right|+\left|\mathbf{Y}^{\mathbf{2}}\right|$ is the total number of cells in $\mathbf{Y}$.

\subsubsection{Sum of row-lengths and squares of row-lengths}

Given a Young diagram $Y$, with rows of length $y_{1} \geqslant y_{2} \geqslant \cdots$, we define

$$
|Y|=\sum_{i=1} y_{i}, \quad\|Y\|=\sum_{i=1} y_{i}^{2}
$$

where the sum is over all parts of $Y .{ }^{4}$ Further, to simplify the equations, we define

$$
|Y|^{\prime}=\frac{1}{2} \sum_{i=1} y_{i}, \quad\|Y\|^{\prime}=\frac{1}{2} \sum_{i=1} y_{i}^{2}
$$

\subsubsection{Sequences}

Given the sequence of row-lengths $\lambda=\left\{\lambda_{1}, \lambda_{2}, \cdots\right\}$, the sequence of half-integers $\rho=$ $\left\{-\frac{1}{2},-\frac{3}{2}, \cdots\right\}$, and two variables $x$ and $y$, we define the 'exponentiated' sequences

$$
x^{-\lambda}=\left\{x^{-\lambda_{1}}, x^{-\lambda_{2}}, \cdots\right\}, \quad y^{-\rho}=\left\{y^{\frac{1}{2}}, y^{\frac{3}{2}}, \cdots\right\}, \quad \text { and } \quad x^{-\lambda} y^{-\rho}=\left\{x^{-\lambda_{1}} y^{\frac{1}{2}}, x^{-\lambda_{2}} y^{\frac{3}{2}}, \cdots\right\}
$$

\footnotetext{
${ }^{4}$ In [9], Iqbal et al. use $\|Y\|^{2}=\sum_{i=1} y_{i}^{2}$. We define $\|Y\|=\sum_{i=1} y_{i}^{2}$ to simplify the notation. This is consistent with other notation and should cause no confusion.
} 


\subsubsection{A function of the arm-lengths and the leg-lengths}

Given a Young diagram $\lambda$, we define the function

$$
Z_{\lambda}[q, t]=\prod_{\square \in \lambda} \frac{1}{\left(1-\left(\frac{q}{t}\right)^{\frac{1}{2}} q^{A_{\square, \lambda}^{+}} t^{L_{\square, \lambda}^{+}}\right)}=\prod_{\square \in \lambda} \frac{1}{\left(1-q^{A_{\square, \lambda}^{++}} t^{L_{\square, \lambda}}\right)}
$$

\subsubsection{Remark}

The expression in the middle of eq. (2.6) corresponds to splitting the cell $\square$ at the corner of a hook in the partition $\lambda$ into two halves, then attaching one half to the arm of that hook to form a half-extended arm of length $A_{\square, \lambda}^{+}=A_{\square, \lambda}+\frac{1}{2}$, and attaching the other half to the leg of that hook to form a half-extended leg of length $L_{\square, \lambda}^{+}=L_{\square, \lambda}+\frac{1}{2}$. The expression on the right corresponds to attaching the cell $\square$ at the corner of a hook to the arm of that hook to form an extended arm of length $A_{\square, \lambda}^{++}=A_{\square, \lambda}+1$. The length of the leg of that hook remains $L_{\square, \lambda}$.

\subsection{Schur and skew-Schur functions}

Given an $n$-row Young diagram $\lambda$, with parts $\lambda_{1} \geqslant \lambda_{2} \geqslant \cdots$, and a set of $n$ variables $\left\{x_{1}, x_{2}, \cdots, x_{n}\right\}$, the Schur function $s_{\lambda}[\mathbf{x}]$ is defined as

$$
s_{\lambda}[\mathbf{x}]=\frac{\operatorname{det}\left(x_{i}^{\lambda_{j}+n-j}\right)_{1 \leqslant i, j \leqslant n}}{\prod_{1 \leqslant i<j \leqslant n}\left(x_{i}-x_{j}\right)}
$$

The skew-Schur function $s_{\lambda / \mu}[\mathbf{x}]$ is defined as

$$
s_{\lambda / \mu}[\mathbf{x}]=\sum_{\nu} c_{\mu \nu}^{\lambda} s_{\lambda}[\mathbf{x}]
$$

where $c_{\mu \nu}^{\lambda}$ are Littlewood-Richardson coefficients defined by

$$
s_{\mu} s_{\nu}=\sum_{\lambda} c_{\mu \nu}^{\lambda} s_{\lambda}
$$

\subsection{Topological vertices}

Our story starts from A-model closed topological string theory on non-compact Calabi-Yau threefolds. We cannot afford to review this vast subject and refer the reader to excellent available introduction, including $[2,3]$.

\subsubsection{The topological vertex of Aganagic et al.}

In [6], Aganagic, Klemm, Marino and Vafa introduced a systematic procedure to calculate A-model topological string partition functions on resolved conifolds. The main ingredient of this procedure is the topological vertex, which has a combinatorial representation in terms of plane partitions with three boundaries specified by three Young diagrams, and can be schematically represented as a trivalent vertex, with bonds labelled by Young diagrams, as in figure 3. For details, we refer to [6]. 


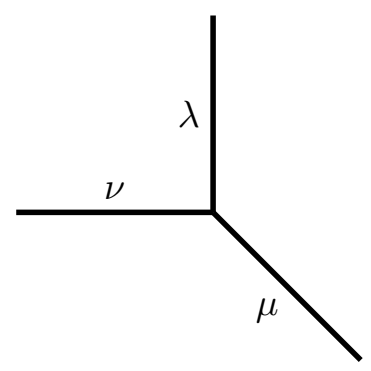

Figure 3. The topological vertex $C_{\lambda \mu \nu}[q, t]$ is trivalent and depends on two parameters $q$ and $t$. The segments are labelled by three partitions $\lambda, \mu$ and $\nu$, such that $\lambda$ is assigned to the vertical segment, $\mu$ is assigned to the segment that follows in a clockwise direction, and $\nu$ to the segment that follows. The 'preferred leg' is labelled by $\nu$.

\subsubsection{The refined topological vertex}

In [9], Iqbal, Kozcaz and Vafa defined the refined topological vertex, up to simple rearrangements, ${ }^{5}$ as

$$
\begin{aligned}
& C_{\lambda \mu \nu}[q, t]= \\
& { }_{q}\left(|\lambda|^{\prime}-|\mu|^{\prime}+\|\mu\|^{\prime}+\|\nu\|^{\prime}\right){ }_{t}\left(-|\lambda|^{\prime}+|\mu|^{\prime}-\left\|\mu^{\top}\right\|^{\prime}\right) \\
& Z_{\nu}[q, t]\left(\sum_{\eta}\left(\frac{q}{t}\right)^{|\eta|^{\prime}} s_{\lambda \tau / \eta}\left[q^{-\nu} t^{-\rho}\right] s_{\mu / \eta}\left[q^{-\rho} t^{-\nu^{\top}}\right]\right)
\end{aligned}
$$

Note that the refined vertex is not manifestly cyclically-symmetric, in the sense that the partitions that label the external legs do not appear on equal footing. In particular, the partition $\nu$ is distinguished from the other two. The external leg labelled by $\nu$ is referred to as 'preferred'. The original, unrefined topological vertex of Aganagic et al. is recovered by setting $q=t$.

\subsubsection{On framing vectors and framing factors}

In defining the refined topological vertex, one labels each of the three boundaries of a vertex by a framing vector that indicates a possible twisting of the boundary. On gluing two vertices along a common boundary, there is in general a framing factor that accounts for a possible mismatch in the orientations of the relevant framing vectors. In this note, we glue vertices such that we do not require framing factors.

\section{A 5D U(2) basic web partition function}

We glue four copies of the refined topological vertex to obtain a 5D basic web that can be used as a building block of $\mathrm{U}(2)$ topological string partition functions.

\subsection{Geometric engineering}

Following $[4,5]$, we write the normalised $\mathrm{U}(2)$ basic web partition function as

$$
\mathcal{W}_{\mathbf{V W} \mathbf{\Delta}}^{\text {norm }}[q, t, R]=\frac{\mathcal{W}_{\mathbf{V W} \mathbf{\Delta}}[q, t, R]}{\mathcal{W}_{\varnothing \varnothing \boldsymbol{\Delta}}[q, t, R]}
$$

\footnotetext{
${ }^{5}$ We use $\mathcal{C}_{\lambda \mu \nu}[q, t]$, and $Z_{Y}[q, t]$, while Iqbal et al. use $\mathcal{C}_{\lambda \mu \nu}[t, q]$, and $\tilde{Z}_{\mu}[t, q]$
} 


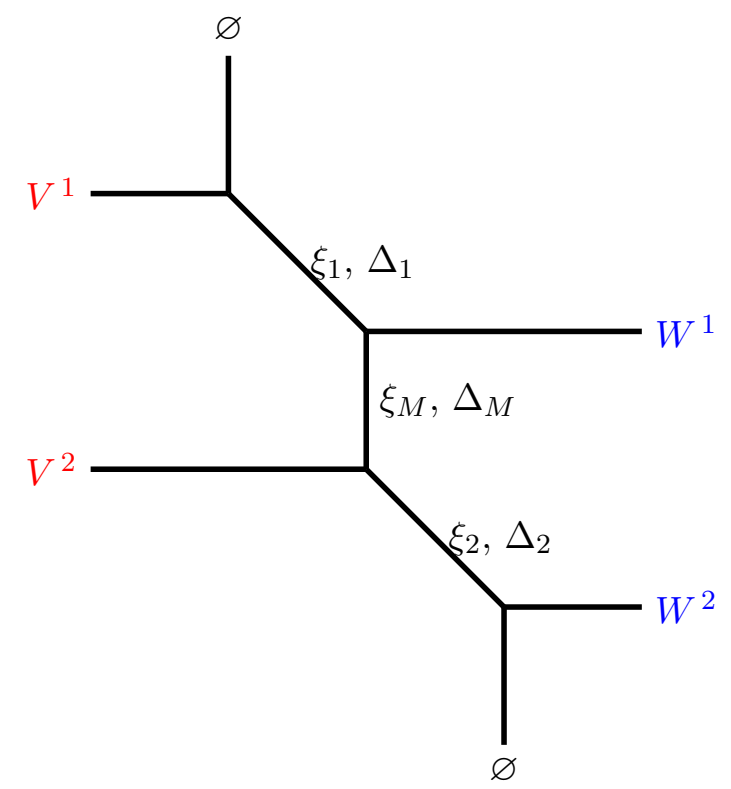

Figure 4. The U(2) basic web diagram. Each external line is labeled by a partition. Each internal line is labeled by a partition and a Kähler parameter. This basic web can be glued to form topological partition functions. The preferred legs are all external, and labelled by the partitions $V^{1}, V^{2}, W^{1}$ and $W^{2}$.

where $\varnothing$ is the trivial, or empty partition with no cells. The numerator is

$$
\begin{aligned}
& \mathcal{W}_{\mathbf{V} \mathbf{W} \boldsymbol{\Delta}}[q, t, R]=\sum_{\xi_{1}, \xi_{M}, \xi_{2}}\left(-Q_{1}\right)^{\left|\xi_{1}\right|}\left(-Q_{M}\right)^{\left|\xi_{M}\right|}\left(-Q_{2}\right)^{\left|\xi_{2}\right|} \\
& \times C_{\varnothing \xi_{1} V^{1}}[q, t] C_{\xi_{M} \xi_{1}^{\top} W^{1 \top}}[t, q] C_{\xi_{M}^{\top} \xi_{2} V^{2}}[q, t] C_{\varnothing \xi_{2}^{\top} W^{2 \top}}[t, q]
\end{aligned}
$$

where we use

$$
Q_{i}=e^{-R \Delta_{i}}, \quad i=1, M, 2
$$

and the denominator $\mathcal{W}_{\varnothing \varnothing \boldsymbol{\Delta}}[q, t, R]$ is identical to the numerator $\mathcal{W}_{\mathbf{V} \mathbf{w} \boldsymbol{\Delta}}[q, t, R]$ but with all external partition pairs empty.

As shown in figure 4, the basic web has two external horizontal legs coming in from the left, two external horizontal legs going out to the right, and a pair of vertical legs, one going up and one down. The horizontal external legs on the left are assigned partitions $\left\{V^{1}, V^{2}\right\}$, the horizontal external legs on the right are assigned partitions $\left\{W^{1}, W^{2}\right\}$. The internal lines are assigned parameters $Q_{1}, Q_{M}$ and $Q_{2}$, and partitions $\xi_{1}$, $\xi_{M}$ and $\xi_{2}$, from top to bottom. The vertical external legs are assigned empty partitions.

Each trivalent vertex corresponds to a refined topological vertex $\mathcal{C}_{\lambda \mu \nu}$. Our convention is such that each vertex has one vertical leg, we associate $\lambda$ to that vertical leg, regardless of whether it is internal or external, pointing upwards or downwards, then $\mu$ and $\nu$ to the remaining two legs, encountered sequentially as we start from the vertical leg and move around the vertex clockwise. Using eq. (2.10), we re-write the numerator in eq. (3.2) as 


$$
\begin{aligned}
& \mathcal{W}_{\mathbf{V W} \boldsymbol{\Delta}}[q, t, R]
\end{aligned}
$$

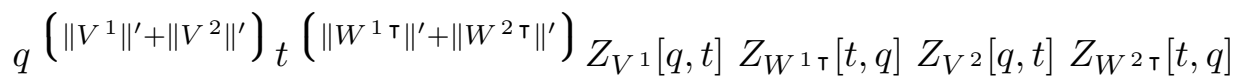

$$
\begin{aligned}
& \times \sum_{\xi_{1}, \xi_{M}, \xi_{2}, \eta_{1}, \eta_{2}}\left(-Q_{1}\right)^{\left|\xi_{1}\right|}\left(-Q_{M}\right)^{\left|\xi_{M}\right|}\left(-Q_{2}\right)^{\left|\xi_{2}\right|}\left(\frac{q}{t}\right)^{\left(\left|\eta_{2}\right|^{\prime}-\left|\eta_{1}\right|^{\prime}\right)} \\
& \times s_{\xi_{1}}\left[q^{-\rho} t^{-V^{1 \top}}\right] s_{\xi_{1}^{\top} / \eta_{1}}\left[q^{-W^{1}} t^{-\rho}\right] s_{\xi_{M}^{\top} / \eta_{1}}\left[q^{-\rho} t^{-W^{1 \top}}\right] \\
& \times s_{\xi_{M} / \eta_{2}}\left[q^{-V^{2}} t^{-\rho}\right] s_{\xi_{2} / \eta_{2}}\left[q^{-\rho} t^{-V^{2} \top}\right] s_{\xi_{2}^{\top}}\left[q^{-W^{2}} t^{-\rho}\right]
\end{aligned}
$$

where we used the fact that for an empty partition $\varnothing$, the skew partition $\varnothing / \eta$ exists only for $\eta=\varnothing$, the sum over $\eta$ trivialises, and the skew Schur function $s_{\varnothing / \eta}=s_{\varnothing}=1$.

\subsubsection{Two skew Schur function identities}

To evaluate the sums in eq. (3.4) for $w^{\text {num }}$, we need the two identities ${ }^{6}$

$$
\begin{aligned}
& \sum_{\lambda} s_{\lambda / \eta_{1}}[x] s_{\lambda / \eta_{2}}[y]=\prod_{i, j}\left(1-x_{i} y_{j}\right)^{-1} \sum_{\tau} s_{\eta_{1} / \tau}[y] s_{\eta_{2} / \tau}[x] \\
& \sum_{\lambda} s_{\lambda / \eta_{1}}[x] s_{\lambda \mathrm{\top} / \eta_{2}}[y]=\prod_{i, j}\left(1+x_{i} y_{j}\right) \sum_{\tau} s_{\eta_{1}^{\top} / \tau^{\top}}[y] s_{\eta_{2}^{\top} / \tau}[x]
\end{aligned}
$$

as well as the property that

$$
Q^{|\lambda|-|\eta|} s_{\lambda / \eta}[x]=s_{\lambda / \eta}[Q x]
$$

which follows from the definition of the skew Schur function.

\subsubsection{The basic web in product form}

We evaluate the sums over the right hand side of eq. (3.4), using $\rho_{i}=-i+\frac{1}{2}$.

\subsubsection{The sums over $\xi_{1}$ and $\xi_{2}$}

$$
\begin{gathered}
\sum_{\xi_{1}}\left(-Q_{1}\right)^{\left|\xi_{1}\right|} s_{\xi_{1}}\left[q^{-\rho} t^{\left.-V^{1 \top}\right]} s_{\xi_{1}^{\top} / \eta_{1}}\left[q^{-W^{1}} t^{-\rho}\right]\right. \\
=\prod_{i, j=1}^{\infty}\left(1-Q_{1} q^{\left.-W_{i}^{1+j-\frac{1}{2}} t^{-V_{j}^{1 \top}+i-\frac{1}{2}}\right) s_{\eta_{1}^{\top}}\left[-Q_{1} q^{-\rho} t^{-V^{1 \top}}\right]}\right. \\
\sum_{\xi_{2}}\left(-Q_{2}\right)^{\left|\xi_{2}\right|} s_{\xi_{2} / \eta_{2}}\left[q^{-\rho} t^{\left.-V^{2 \top}\right]} s_{\xi_{2}^{\top}}\left[q^{-W^{2}} t^{-\rho}\right]\right. \\
=\prod_{i, j=1}^{\infty}\left(1-Q_{2} q^{-W_{i}^{2}+j-\frac{1}{2}} t^{-V_{j}^{2 \top}+i-\frac{1}{2}}\right) s_{\eta_{2}^{\top}}\left[-Q_{2} q^{-W^{2}} t^{-\rho}\right]
\end{gathered}
$$

\footnotetext{
${ }^{6}$ Exercise 26, page 93 of [23].
} 


\subsubsection{The sum over $\xi_{M}$}

We re-write this in terms of a sum over a new set of partition $\tau$,

$$
\begin{aligned}
& \sum_{\xi_{M}}\left(-Q_{M}\right)^{\left|\xi_{M}\right|-\left|\eta_{1}\right|} s_{\xi_{M}^{\top} / \eta_{1}}\left[q^{-\rho} t^{\left.-W^{1, \top}\right]} s_{\xi_{M} / \eta_{2}}\left[q^{-V^{2}} t^{-\rho}\right]\right. \\
& =\prod_{i, j}\left(1-Q_{M} q^{-V_{i}{ }^{2}+j-\frac{1}{2}} t^{-W_{j}^{1 \top}+i-\frac{1}{2}}\right) \sum_{\tau} s_{\eta_{1}^{\top} / \tau^{\top}}\left[q^{-V^{2}} t^{-\rho}\right] s_{\eta_{2}^{\top} / \tau}\left[-Q_{M} q^{-\rho} t^{-W^{1 \top}}\right]
\end{aligned}
$$

\subsubsection{The sums over $\eta_{1}$ and $\eta_{2}$}

$$
\begin{gathered}
\sum_{\eta_{1}}\left(\frac{q}{t}\right)^{-\left|\eta_{1}\right|^{\prime}}\left(-Q_{M}\right)^{\left|\eta_{1}\right|} s_{\eta_{1}^{\top}}\left[-Q_{1} q^{-\rho} t^{\left.-V^{1 \top}\right]} s_{\eta_{1}^{\top} / \tau^{\top}}\left[q^{-V^{2}} t^{-\rho}\right]\right. \\
=\prod_{i, j}\left(1-Q_{1} Q_{M} q^{-V_{i}{ }^{2}+j-1} t^{-V_{j}^{1 \top}+i}\right)^{-1} s_{\tau^{\top}}\left[Q_{1} Q_{M} q^{j-\frac{1}{2}} t^{\left.-V_{j}^{1 \top}\right]}\right] \\
\sum_{\eta_{2}}\left(\frac{q}{t}\right)^{\left|\eta_{2}\right|^{\prime}} s_{\eta_{2}^{\top}}\left[-Q_{2} q^{-W^{2}} t^{-\rho}\right] s_{\eta_{2}^{\top} / \tau}\left[-Q_{M} q^{-\rho} t^{\left.-W^{1 \top}\right]}\right. \\
=\prod_{i, j}\left(1-Q_{M} Q_{2} q^{-W_{i}^{2}+j} t^{-W_{j}^{1}+i-1}\right)^{-1} s_{\tau}\left[-Q_{2} q^{-W_{i}{ }^{2}} t^{i-\frac{1}{2}}\right]
\end{gathered}
$$

\subsubsection{The sum over $\tau$}

We finally evaluate the sum over the partitions that were introduced in an intermediate step above,

$$
\sum_{\tau} s_{\tau}\left[-Q_{2} q^{-W_{i}^{2}} t^{i-\frac{1}{2}}\right] s_{\tau \top}\left[Q_{1} Q_{M} q^{j-\frac{1}{2}} t^{-V_{j}^{1 \top}}\right]=\prod_{i, j}\left(1-Q_{1} Q_{M} Q_{2} q^{-W_{i}^{2}+j-\frac{1}{2}} t^{-V_{j}{ }^{1}+i-\frac{1}{2}}\right)
$$

to obtain

$$
\begin{aligned}
& \mathcal{W}_{\mathbf{V W} \boldsymbol{\Delta}}[q, t, R]= \\
& q\left(\left\|V^{1}\right\|^{\prime}+\left\|V^{2}\right\|^{\prime}\right)_{t}\left(\left\|W^{1 \top}\right\|^{\prime}+\left\|W^{2 \top}\right\|^{\prime}\right) Z_{V^{1}}[q, t] Z_{W^{1 \top}}[t, q] Z_{V^{2}}[q, t] Z_{W^{2 \top}}[t, q] \\
& \times \prod_{i, j=1}^{\infty}\left(1-Q_{1} q^{-W_{i}{ }^{1}+j-\frac{1}{2}} t^{-V_{j}^{1 \top}+i-\frac{1}{2}}\right) \prod_{i, j=1}^{\infty}\left(1-Q_{2} q^{-W_{i}^{2}+j-\frac{1}{2}} t^{-V_{j}^{2}{ }^{2}+i-\frac{1}{2}}\right) \\
& \times \prod_{i, j=1}^{\infty}\left(1-Q_{M} q^{-V_{i}^{2}+j-\frac{1}{2}} t^{-W_{j}^{1 \top}+i-\frac{1}{2}}\right) \prod_{i, j=1}^{\infty}\left(1-Q_{1} Q_{M} Q_{2} q^{-W_{i}^{2}+j-\frac{1}{2}} t^{-V_{j}^{1 \top}+i-\frac{1}{2}}\right) \\
& \times \prod_{i, j=1}^{\infty}\left(1-Q_{1} Q_{M} q^{-V_{i}^{2}+j-1} t^{-V_{j}^{1 \top}+i}\right)^{-1} \prod_{i, j=1}^{\infty}\left(1-Q_{M} Q_{2} q^{-W_{i}^{2}+j} t^{-W_{j}^{1 \top}+i-1}\right)^{-1}
\end{aligned}
$$

Note that regarding the right hand side of eq. (3.14) as a rational function, the initial four products are in the numerator, while the latter two are in the denominator. 


\subsubsection{Normalised products}

Given two partitions, $V$ with parts $v_{i}, i=1,2, \cdots$, and $W$ with parts $w_{i}, i=1,2, \cdots$, and two sequences of integers $\alpha_{k}$ and $\beta_{k}, k=1,2, \cdots$, we define

$$
\prod_{i, j=1}^{\infty}\left(1-Q q^{-v_{i}+\alpha_{j}} t^{-w_{j}+\beta_{i}}\right)=\prod_{i, j=1}^{\infty}\left(\frac{1-Q q^{-v_{i}+\alpha_{j}} t^{-w_{j}+\beta_{i}}}{1-Q q^{\alpha_{i}} t^{\beta_{i}}}\right)
$$

In this notation, the expression for $\mathcal{W}_{\mathbf{V W} \mathbf{~} \mathbf{\Delta}}^{\text {norm }}[q, t, R]$ in eq. (3.1), is identical to that for $\mathcal{W}_{\mathbf{V W} \boldsymbol{\Delta}}[q, t, R]$, in eq. (3.4), up to replacing each product $\prod_{i, j}$ by a normalised product $\prod_{i, j}^{\prime}$.

\subsubsection{From infinite to finite products}

Using eq. (3.15), we have the following identities $[8,24] .{ }^{7}$ Firstly,

$$
\begin{gathered}
\prod_{i, j=1}^{\infty}\left(1-Q q^{-w_{i}+j-1} t^{-v_{j}^{\top}+i}\right)=\prod_{\square \in V}\left(1-Q q^{-A_{\square, V}^{++}} t^{-L_{\square, W}}\right) \prod_{\square \in W}\left(1-Q q^{A} \boldsymbol{\bullet , W} t^{L_{\mathbf{m}, V}^{++}}\right) \\
=\prod_{\square \in W}\left(1-Q q^{-A_{\mathbf{\bullet}, V}^{++}} t^{-L_{\mathbf{\bullet}, W}}\right) \prod_{\square \in V}\left(1-Q q^{A_{\square, W}} t^{L_{\square, V}^{++}}\right)
\end{gathered}
$$

Note that while the product on the left of eq. (3.16) is normalised in the sense of eq. (3.15), the remaining products are not.

\subsubsection{The $5 \mathrm{D}$ basic web in product form}

Using the identities (3.16) in eq. (3.4) for the numerator, we write the normalised $\mathrm{U}(2)$ basic web partition function in eq. (3.1) as

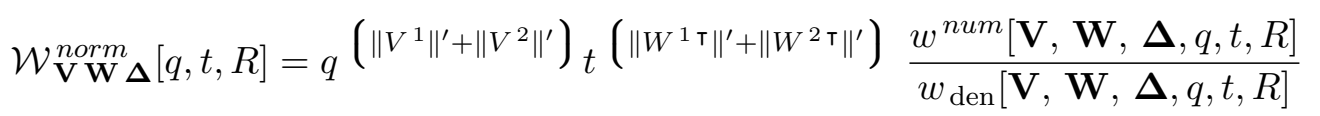

where, using $Q_{3}=Q_{1} Q_{M} Q_{2}, w^{n u m}$ is

$$
\begin{aligned}
& w^{n u m}[\mathbf{V}, \mathbf{W}, \boldsymbol{\Delta}, q, t, R]=\prod_{\square \in V^{1}}\left(1-Q_{1} q^{-A_{\square, W^{1}}^{+}} t^{-L_{\square, V^{1}}^{+}}\right) \prod_{\mathbf{⿴} \in W^{1}}\left(1-Q_{1} q^{A_{\mathbf{\bullet}^{+} V^{1}}^{1}} t^{L_{\mathbf{\bullet}, W^{1}}^{+}}\right) \\
& \times \prod_{\square \in V^{2}}\left(1-Q_{2} q^{-A_{\square, W^{2}}^{+}} t^{-L_{\square, V^{2}}^{+}}\right) \prod_{\mathbf{\square} \in W^{2}}\left(1-Q_{2} q^{A_{\mathbf{\bullet}_{,} V^{2}}^{+} t^{L_{\mathbf{\bullet}, W^{2}}^{+}}}\right) \\
& \times \prod_{\square \in W^{1}}\left(1-Q_{M} q^{-A_{\bullet}^{+}, V^{2}} t^{-L_{\mathbf{\bullet}, W^{1}}^{+}}\right) \prod_{\square \in V^{2}}\left(1-Q_{M} q^{A_{\square, W^{1}}^{+}} t^{L_{\square, V^{2}}^{+}}\right) \\
& \times \prod_{\square \in V^{1}}\left(1-Q_{3} q^{-A_{\square, W^{2}}^{+}} t^{-L_{\square, V^{1}}^{+}}\right) \prod_{\square \in W^{2}}\left(1-Q_{3} q^{A_{\boldsymbol{\bullet}, V^{1}}^{+}} t^{L_{\mathbf{\bullet}, W^{2}}^{+}}\right),
\end{aligned}
$$

where we have used the second equality in eq. (3.16) to put the products in the above uniform form. The denominator $w_{\text {den }}$ is

\footnotetext{
${ }^{7}$ For an excellent reference and compendium of relevant combinatorial identities, including proofs, see [8]. Eq. (3.16) in this note follows from eqs. 2.8-2.11 in [8].
} 


$$
\begin{aligned}
& w_{\operatorname{den}}[\mathbf{V}, \mathbf{W}, \boldsymbol{\Delta}, q, t, R]=\prod_{\square \in V^{1}}\left(1-q^{A_{\square, V^{1}}^{++}} t^{L_{\square, V^{1}}}\right) \prod_{\square \in W^{1}}\left(1-q^{A} \mathbf{\varpi}^{1} W^{1} t^{L_{\boldsymbol{\bullet}^{+}, W^{1}}^{+}}\right) \\
& \times \prod_{\square \in V^{2}}\left(1-q^{A_{\square, V^{2}}^{++}} t^{L_{\square, V^{2}}}\right) \prod_{\square \in W^{2}}\left(1-q^{A} \mathbf{\square}_{W^{2}} t^{L_{\mathbf{\varpi}^{+}, W^{2}}^{++}}\right) \\
& \times \prod_{\square \in V^{1}}\left(1-Q_{1} Q_{M} q^{-A_{\square, V^{2}}^{++}} t^{-L_{\square, V^{1}}}\right) \prod_{\square \in V^{2}}\left(1-Q_{1} Q_{M} q^{A_{\square, V^{1}}} t^{L_{\square, V^{2}}^{++}}\right)
\end{aligned}
$$

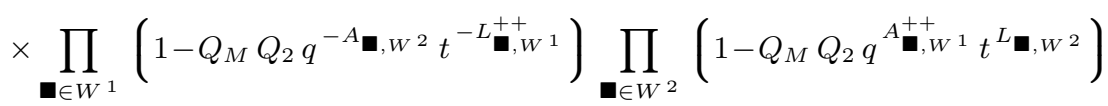

where the first four products on the right hand side of eq. (3.19) are due to the product $Z_{V^{1}}[t, q] Z_{W^{1 \top}}[q, t] Z_{V^{2}}[t, q] Z_{W^{2 \top}}[q, t]$ in eq. (3.4). $w_{\text {den }}$ is equal to the denominator $\mathcal{W}_{\varnothing \varnothing \Delta}[q, t, R]$ on the right hand side of eq. (3.1).

\subsubsection{Remark}

One can glue copies of the basic web partition function $\mathcal{W}_{\mathbf{V} \mathbf{~ W} \boldsymbol{\Delta}}[q, t, R]$ in several ways. In this note, we restrict our attention to gluing linearly or cyclically, to form linear or cyclic $\mathrm{U}(2)$ quiver gauge theories, as described in paragraphs 7.1 and 7.2. We do not, for example, glue basic webs to form a Hirzbruch surface.

\section{A 4D U(2) basic web partition function}

We take the $R \rightarrow 0$ limit of the $5 D$ basic web partition function to obtain its $4 D$ analogue.

\subsection{Two parameters}

We take the relationship between the parameters $q$ and $t$ of the refined vertex and the parameters $\epsilon_{1}$ and $\epsilon_{2}$ of the instanton partition function to be,

$$
q=e^{R \epsilon_{2}}, \quad t=e^{-R \epsilon_{1}}
$$

where $R$, the radius of a space-like circle, plays the role of a deformation parameter. We write $\mathcal{W}_{\mathbf{V W} \mathbf{\Delta}}^{\text {norm }}\left[\epsilon_{1}, \epsilon_{2}, R\right]$, then take the limit $R \rightarrow 0$. The prefactor on the left hand side of eq. (3.17) tends to 1 in the limit $R \rightarrow 0$, and we obtain

$$
\mathcal{W}_{\mathbf{V W} \mathbf{\Delta}}^{\text {norm }}\left[\epsilon_{1}, \epsilon_{2}, R \rightarrow 0\right]=\frac{w^{\text {num }}\left[\mathbf{V}, \mathbf{W}, \boldsymbol{\Delta}, \epsilon_{1}, \epsilon_{2}, R \rightarrow 0\right]}{w_{\operatorname{den}}\left[\mathbf{V}, \mathbf{W}, \boldsymbol{\Delta}, \epsilon_{1}, \epsilon_{2}, R \rightarrow 0\right]}
$$

where, using $\Delta_{3}=\Delta_{1}+\Delta_{M}+\Delta_{2}$, we have

$$
\begin{aligned}
w^{n u m} & {\left[\mathbf{V}, \mathbf{W}, \boldsymbol{\Delta}, \epsilon_{1}, \epsilon_{2}, R \rightarrow 0\right]=} \\
& \prod_{\square \in V^{1}}\left(\Delta_{1}+A_{\square, W^{1}}^{+} \epsilon_{2}-L_{\square, V^{1}}^{+} \epsilon_{1}\right) \prod_{\mathbf{\square} \in W^{1}}\left(\Delta_{1}-A_{\square, V^{1}}^{+} \epsilon_{2}+L_{\mathbf{\square}, W^{1}}^{+} \epsilon_{1}\right) \\
& \times \prod_{\square \in V^{2}}\left(\Delta_{2}+A_{\square, W^{2}}^{+} \epsilon_{2}-L_{\square, V^{2}}^{+} \epsilon_{1}\right) \prod_{\square \in W^{2}}\left(\Delta_{2}-A_{\square, V^{2}}^{+} \epsilon_{2}+L_{\square}^{+}, W^{2} \epsilon_{1}\right) \\
& \times \prod_{\square \in V^{2}}\left(\Delta_{M}-A_{\square, W^{1}}^{+} \epsilon_{2}+L_{\square, V^{2}}^{+} \epsilon_{1}\right) \prod_{\square \in W^{1}}\left(\Delta_{M}+A_{\square, V^{2}}^{+} \epsilon_{2}-L_{\mathbf{\square}, W^{1}}^{+} \epsilon_{1}\right)
\end{aligned}
$$




$$
\times \prod_{\square \in V^{1}}\left(\Delta_{3}+A_{\square, W^{2}}^{+} \epsilon_{2}-L_{\square, V^{1}}^{+} \epsilon_{1}\right) \prod_{\square \in W^{2}}\left(\Delta_{3}-A_{\square}^{+}, V^{1} \epsilon_{2}+L_{\mathbf{\square}, W^{2}}^{+} \epsilon_{1}\right)
$$

and

$$
\begin{gathered}
w_{\operatorname{den}}\left[\mathbf{V}, \mathbf{W}, \boldsymbol{\Delta}, \epsilon_{1}, \epsilon_{2}, R \rightarrow 0\right]= \\
\prod_{\square \in V^{1}}\left(-A_{\square, V^{1}}^{++} \epsilon_{2}+L_{\square, V^{1}} \epsilon_{1}\right) \prod_{\square \in V^{2}}\left(-A_{\square, V^{2}}^{++} \epsilon_{2}+L_{\square, V^{2}} \epsilon_{1}\right) \\
\times \prod_{\square \in W^{1}}\left(-A_{\square, W^{1}} \epsilon_{2}+L_{\square, W^{1}}^{++} \epsilon_{1}\right) \prod_{\square \in W^{2}}\left(-A_{\square, W^{2}} \epsilon_{2}+L_{\square, W^{2}}^{++} \epsilon_{1}\right) \\
\times \prod_{\square \in V^{1}}\left(\Delta_{1}+\Delta_{M}+A_{\square, V^{2}}^{++} \epsilon_{2}-L_{\square, V^{1}} \epsilon_{1}\right) \prod_{\square \in V^{2}}\left(\Delta_{1}+\Delta_{M}-A_{\square, V^{1}} \epsilon_{2}+L_{\square, V^{2}}^{++} \epsilon_{1}\right) \\
\times \prod_{\square \in W^{1}}\left(\Delta_{2}+\Delta_{M}+A_{\square, W^{2}} \epsilon_{2}-L_{\square, W^{1}}^{++} \epsilon_{1}\right) \prod_{\square \in W^{2}}\left(\Delta_{2}+\Delta_{M}-A_{\square, W^{1}}^{++} \epsilon_{2}+L_{\mathbf{\square}, W^{2}} \epsilon_{1}\right)
\end{gathered}
$$

\section{The building block of the $4 \mathrm{D} \mathrm{U}(2)$ quiver instanton partition function}

We recall the normalised contribution of the bifundamental hypermultiplet which acts as a building block of the instanton partition function.

In the notation of eq. 6 and section 2 of [17], the normalised bifundamental partition function $\mathcal{Z}_{\text {building.block }}^{4 \mathrm{D}}$ is

$$
\mathcal{Z}_{\text {building.block }}^{4 \mathrm{D}}\left(\mathbf{a}, \mathbf{V}^{\prime}|\mu| \mathbf{b}, \mathbf{W}^{\prime}\right)=\frac{z^{\text {num }}\left(\mathbf{a}, \mathbf{V}^{\prime}|\mu| \mathbf{b}, \mathbf{W}^{\prime}\right)}{z_{\text {den }}\left(\mathbf{a}, \mathbf{V}^{\prime} \mid \mathbf{b}, \mathbf{W}^{\prime}\right)}
$$

where $\mathbf{a}=\{a,-a\}, \mathbf{b}=\{b,-b\}, a, b$ and $\mu$ are linear combinations of $\epsilon_{1}$ and $\epsilon_{2}$, as will be explained in section 8 below, and we use $\mathbf{V}^{\prime}$ and $\mathbf{W}^{\prime}$ for partition pairs that we will relate in section 7 to the pairs that appear in the $4 \mathrm{D}$ basic web. We refer to [17] for brief explanations of the parameters that appear in $\mathcal{Z}_{\text {building.block. Defining }}^{4 \mathrm{D}}$

$$
\alpha_{0}=\frac{1}{2}\left(\epsilon_{1}+\epsilon_{2}\right)
$$

the numerator $z^{\text {num }}$, as given in eq. 9 of [17], is

$$
\begin{aligned}
& z^{\text {num }}\left(\mathbf{a}, \mathbf{V}^{\prime}|\mu| \mathbf{b}, \mathbf{W}^{\prime}\right)= \\
& \prod_{\square \in V^{1}}\left(\left[a-b-\mu+\alpha_{0}\right]+A_{\square, V^{1}}^{+}, \epsilon_{1}-L_{\square, W^{1}}^{+}, \epsilon_{2}\right) \prod_{\square \in W^{1},}\left(\left[a-b-\mu+\alpha_{0}\right]-A_{\square, W^{1}}^{+}, \epsilon_{1}+L_{\mathbf{\square}, V^{1}}^{+}, \epsilon_{2}\right) \\
& \times \prod_{\square \in V^{2},}\left(\left[-a+b-\mu+\alpha_{0}\right]+A_{\square, V^{2}}^{+}, \epsilon_{1}-L_{\square, W^{2}}^{+}, \epsilon_{2}\right) \prod_{\square \in W^{2},}\left(\left[-a+b-\mu+\alpha_{0}\right]-A_{\square}^{+}, W^{2}, \epsilon_{1}+L_{\square, V^{2}}^{+}, \epsilon_{2}\right) \\
& \times \prod_{\square \in V^{2},}\left(\left[-a-b-\mu+\alpha_{0}\right]+A_{\square, V^{2}}^{+}, \epsilon_{1}-L_{\square, W^{1}}^{+}, \epsilon_{2}\right) \prod_{\square \in W^{1},}\left(\left[-a-b-\mu+\alpha_{0}\right]-A_{\square, W^{1}}^{+}, \epsilon_{1}+L_{\square}^{+}, V^{2}, \epsilon_{2}\right) \\
& \times \prod_{\square \in V^{1}}\left(\left[a+b-\mu+\alpha_{0}\right]+A_{\square, V^{1}}^{+}, \epsilon_{1}-L_{\square, W^{2}}^{+}, \epsilon_{2}\right) \prod_{\square \in W^{2},}\left(\left[a+b-\mu+\alpha_{0}\right]-A_{\square}^{+}, W^{2}, \epsilon_{1}+L_{\square}^{+}, V^{1}, \epsilon_{2}\right)
\end{aligned}
$$


The denominator $z_{\text {den }}$, as given in eq. 7 of [17], is

$$
z_{\operatorname{den}}\left(\mathbf{a}, \mathbf{V}^{\prime} \mid \mathbf{b}, \mathbf{W}^{\prime}\right)=\left(z^{\text {num }}\left(\mathbf{a}, \mathbf{V}^{\prime}|0| \mathbf{a}, \mathbf{V}^{\prime}\right) z^{\text {num }}\left(\mathbf{b}, \mathbf{W}^{\prime}|0| \mathbf{b}, \mathbf{W}^{\prime}\right)\right)^{\frac{1}{2}}
$$

\section{Identification of $\mathcal{W}_{\mathrm{VW} \Delta}^{\text {norm }}$ and $\mathcal{Z}_{\text {building.block }}^{4 \mathrm{D}}$. The numerators}

Comparing eq. (4.3) and eq. (5.3), we find that if we set $V^{i \prime}=V^{i \top}, W^{i \prime}=W^{i \top}$, and multiply each factor by -1 , which is possible since the number of factors is even by construction, we obtain

$$
\begin{aligned}
& z^{\text {num }}(\mathbf{a}, \mathbf{V}|\mu| \mathbf{b}, \mathbf{W})= \\
& \prod_{\square \in V^{1}}\left(\left[-a+b+\mu-\alpha_{0}\right]+A_{\square, W^{1}}^{+} \epsilon_{2}-L_{\square, V^{1}}^{+} \epsilon_{1}\right) \prod_{\square \in W^{1}}\left(\left[-a+b+\mu-\alpha_{0}\right]-A_{\square, V^{1}}^{+} \epsilon_{2}+L_{\square, W^{1}}^{+} \epsilon_{1}\right) \\
& \times \prod_{\square \in V^{2}}\left(\left[a-b+\mu-\alpha_{0}\right]+A_{\square, W^{2}}^{+} \epsilon_{2}-L_{\square, V^{2}}^{+} \epsilon_{1}\right) \prod_{\square \in W^{2}}\left(\left[a-b+\mu-\alpha_{0}\right]-A_{\square}^{+} V_{V^{2}} \epsilon_{2}+L_{\square, W^{2}}^{+} \epsilon_{1}\right) \\
& \times \prod_{\square \in V^{2}}\left(\left[a+b+\mu-\alpha_{0}\right]+A_{\square, W^{1}}^{+} \epsilon_{2}-L_{\square, V^{2}}^{+} \epsilon_{1}\right) \prod_{\square \in W^{1}}\left(\left[a+b+\mu-\alpha_{0}\right]-A_{\square}^{+},_{V^{2}} \epsilon_{2}+L_{\square}^{+}, W^{1} \epsilon_{1}\right) \\
& \times \prod_{\square \in V^{1}}\left(\left[-a-b+\mu-\alpha_{0}\right]+A_{\square, W^{2}}^{+} \epsilon_{2}-L_{\square, V^{1}}^{+} \epsilon_{1}\right) \prod_{\square \in W^{2}}\left(\left[-a-b+\mu-\alpha_{0}\right]-A_{\square, V^{1}}^{+} \epsilon_{2}+L_{\square}^{+}, W^{2} \epsilon_{1}\right)
\end{aligned}
$$

which leads to the identification

$$
\Delta_{1}=-a+b+\mu-\alpha_{0}, \quad \Delta_{2}=a-b+\mu-\alpha_{0}, \quad \Delta_{M}=-a-b-\mu+\alpha_{0}
$$

\section{Identification of $\mathcal{W}_{\mathrm{VW} \Delta}^{\text {norm }}$ and $\mathcal{Z}_{\text {building.block }}^{4 \mathrm{D}}$. The denominators}

Using the identification of parameters obtained in eq. (6.2) in $w_{\text {den }}[\mathbf{V}, \mathbf{W}, \boldsymbol{\Delta}]$ and $z_{\text {den }}$ as given in eqs. (4.4) and (5.4), it is clear that these two functions are not the same. However, what matters is not the denominator of s single factor, but the product of all denominators, as we explain below.

The denominator $w_{\text {den }}[\mathbf{V}, \mathbf{W}, \boldsymbol{\Delta}]$ is a natural object, as we can see in the derivation in section 3. On the other hand, the denominator $z_{\text {den }}$ was obtained in [17] by taking the full denominator that appears in expressions for the $4 \mathrm{D} \mathrm{U}(1)$ linear and cyclic quiver instanton partition functions and factoring that into denominators for the contributions of the bifundamental hypermultiplets. Such a factorisation is not unique and any factorisation is allowed for as long as the product of all factors is equal to the full denominator of the original expression.

In this work, to identify $\mathcal{W}^{4 \mathrm{D}}$ and $\mathcal{Z}_{\text {building.block }}^{4 \mathrm{D}}$, we need a factor $\mathcal{F}$, such that the product of all normalisation factors that appear in a conformal block is equal to 1 . Consider the abbreviations

$$
\mathcal{A}_{V^{i j}}[x]=\mathcal{A}_{V^{i}, V^{j}}[x]=\prod_{\square \in V^{i}}\left(x+A_{\square, V^{i}}^{++} \epsilon_{2}-L_{\square, V^{j}} \epsilon_{1}\right), \quad \mathcal{A}_{\varnothing, \varnothing}[x]=1,
$$




$$
\begin{aligned}
\mathcal{L}_{W^{i j}}[x]=\mathcal{L}_{W^{i}, W^{j}}[x] & =\prod_{\mathbf{\square} \in W^{j}}\left(x-A_{\mathbf{m}, W^{j}} \epsilon_{2}+L_{\mathbf{\square}, W^{i}}^{++} \epsilon_{1}\right), \quad \mathcal{L}_{\varnothing, \varnothing}[x]=1, \\
\mathcal{H}_{Y^{i j}}[x] & =\mathcal{A}_{Y^{i j}}[x] \mathcal{L}_{Y^{i j}}[x]
\end{aligned}
$$

In this notation, $z_{\text {den }}$ and $w_{\text {den }}$ are

$$
\begin{aligned}
& z_{\text {den }}(\mathbf{a}, \mathbf{V}|0| \mathbf{b}, \mathbf{W})= \\
& \left(\mathcal{H}_{V^{11}}[0] \mathcal{H}_{V^{12}}[2 a] \mathcal{H}_{V^{12}[-2 a]} \mathcal{H}_{V^{22}}[0] \mathcal{H}_{W^{11}}[0] \mathcal{H}_{W^{12}}[2 b] \mathcal{H}_{W^{12}}[-2 b] \mathcal{H}_{W^{22}}[0]\right)^{\frac{1}{2}}
\end{aligned}
$$

and

$$
\begin{aligned}
& w_{\operatorname{den}}\left[\mathbf{V}, \mathbf{W}, \boldsymbol{\Delta}, \epsilon_{1}, \epsilon_{2}, R \rightarrow 0\right]=w_{\operatorname{den}}(\mathbf{a}, \mathbf{V} \mid \mathbf{b}, \mathbf{W})= \\
& \quad(-)^{\left|V^{1}\right|+\left|V^{2}\right|+\left|W^{1}\right|+\left|W^{2}\right|} \mathcal{L}_{V^{11}}[0] \mathcal{L}_{V^{22}}[0] \mathcal{H}_{V^{12}}[-2 a] \mathcal{H}_{W^{12}}[2 b] \mathcal{A}_{W^{11}}[0] \mathcal{A}_{W^{22}}[0]
\end{aligned}
$$

Now consider the factor

$$
\mathcal{F}(\mathbf{a}, \mathbf{V} \mid \mathbf{b}, \mathbf{W})=\frac{z_{\operatorname{den}}[\mathbf{a}, \mathbf{V} \mid \mathbf{b}, \mathbf{W}]}{w_{\operatorname{den}}[\mathbf{a}, \mathbf{V} \mid \mathbf{b}, \mathbf{W}]}
$$

and define

$$
z_{\text {den }}^{\prime}=\mathcal{F}^{-1} z_{\text {den }}=w_{\text {den }}, \quad \mathcal{Z}_{\text {building.block }}^{\prime}=\mathcal{F} \mathcal{Z}_{\text {building.block }}
$$

$\mathcal{Z}_{\text {building.block }}^{\prime}$ is constructed such that 1 . It has the same numerator as $\mathcal{Z}_{\text {building.block }}^{4 \mathrm{D}}$, which is the same as that of $\mathcal{W}_{\mathbf{V W} \boldsymbol{\Delta}}^{\text {norm }}$, when we choose the parameters as in eq. (6.2) and $\mathbf{2}$. It has the same denominator as $\mathcal{W}_{\mathbf{V W} \boldsymbol{\Delta}}^{\text {norm }}$, also when we choose the parameters as in eq. (6.2).

Since the denominator of $\mathcal{Z}_{\text {building.block }}^{\prime}$ is not manifestly the same as that of $\mathcal{Z}_{\text {building.block}}^{4 D}$, we need to show that gluing copies of $\mathcal{Z}_{\text {building.block }}^{\prime}$ to build a topological partition function, leads to the same result obtained by gluing copies of the original $\mathcal{Z}_{\text {building.block. }} \mathcal{F}$ can be written in a simpler form as follows,

where

$$
(\mathcal{F}(\mathbf{a}, \mathbf{V} \mid \mathbf{b}, \mathbf{W}))^{2}=F_{\mathbf{V}}^{\text {left }}[a] F_{\mathbf{W}}^{\text {right }}[b]
$$

$$
\begin{aligned}
F_{\mathbf{V}}^{\text {left }}[a] & =(-)^{\left|V^{1}\right|+\left|V^{2}\right|}\left(\frac{\mathcal{A}_{V^{11}}[0] \mathcal{A}_{V^{22}}[0]}{\mathcal{L}_{V^{11}}[0] \mathcal{L}_{V^{22}}[0]}\right)\left(\frac{\mathcal{H}_{V^{12}}[2 a]}{\mathcal{H}_{V^{12}}[-2 a]}\right) \\
F_{\mathbf{W}}^{\text {right }}[b] & =(-)^{\left|W^{1}\right|+\left|W^{2}\right|}\left(\frac{\mathcal{H}_{W^{12}}[-2 b]}{\mathcal{H}_{W^{12}}[2 b]}\right)\left(\frac{\mathcal{L}_{W^{11}}[0] \mathcal{L}_{W^{22}}[0]}{\mathcal{A}_{W^{11}}[0] \mathcal{A}_{W^{22}}[0]}\right)
\end{aligned}
$$

$F^{\text {right }}$ and $F^{\text {left }}$ satisfy the obvious properties

$$
F_{\varnothing}^{\text {right }}[x]=F_{\varnothing}^{\text {left }}[x]=1
$$

and

$$
F_{\mathbf{Y}}^{\text {right }}[x] F_{\mathbf{Y}}^{\text {left }}[x]=1
$$

The physical objects that we are interested in are the conformal blocks which are constructed by gluing copies of $\mathcal{Z}_{\text {building.block }}$ [17]. We need to show that gluing copies of $\mathcal{Z}_{\text {building.block }}^{\prime}$ leads to the same result, which will be the case if products of the normalisation factors trivialise. This will follow directly from eqs. (7.9) and (7.10). There are two cases to consider, the linear conformal block case and the cyclic conformal block case. 


\subsection{Linear conformal blocks}

Consider the linear conformal block obtained by gluing $n$ copies of $\mathcal{Z}_{\text {building.block, that }}$ is $\mathcal{Z}_{\text {building.block.1 }}, \mathcal{Z}_{\text {building.block.2 }}, \cdots, \mathcal{Z}_{\text {building.block.n, }}$ sequentially. Using copies of $\mathcal{Z}_{\text {building.block}}^{\prime}$, we obtain the same result as using copies of $\mathcal{Z}_{\text {building.block }}$ up to a factor

$$
F_{\varnothing}^{\text {left }}\left[x_{0}\right] F_{\mathbf{Y}^{1}}^{\text {right }}\left[x_{1}\right] F_{\mathbf{Y}^{1}}^{\text {left }}\left[x_{1}\right] F_{\mathbf{Y}^{\mathbf{2}}}^{\text {right }}\left[x_{2}\right] \cdots F_{\mathbf{Y}^{\mathbf{n}-1}}^{\text {left }}\left[x_{n-1}\right] F_{\varnothing}^{\text {right }}\left[x_{n}\right]=1
$$

\subsection{Cyclic conformal blocks}

$$
F_{\mathbf{Y}^{\mathbf{0}}}^{\text {left }}\left[x_{0}\right] F_{\mathbf{Y}^{\mathbf{1}}}^{\mathrm{right}}\left[x_{1}\right] F_{\mathbf{Y}^{1}}^{\text {left }}\left[x_{1}\right] F_{\mathbf{Y}^{\mathbf{2}}}^{\mathrm{right}}\left[x_{2}\right] \cdots F_{\mathbf{Y}^{\mathbf{n}-\mathbf{1}}}^{\text {left }}\left[x_{n-1}\right] F_{\mathbf{Y}^{\mathbf{0}}}^{\mathrm{right}}\left[x_{0}\right]=1
$$

We conclude that $\mathcal{Z}_{\text {building.block }}^{\prime}$ leads to the same conformal blocks as $\mathcal{Z}_{\text {building.block. }}$

\subsection{The denominator $z_{\text {den }}^{\prime}$ and the Burge conditions}

In $[16,17]$, it was shown that for the choice of parameters that leads to Virasoro $A$-series minimal conformal blocks, the denominator $z_{\text {den }}$ will contain non-physical zeros, unless we restrict the partition pairs, that $\mathcal{Z}_{\text {building.block }}^{4 \mathrm{D} \text {, min }}$ depends on, to obey Burge conditions. These conditions were derived in [17] using $z_{\text {den }}$ rather than $z_{\text {den }}^{\prime}$. Using $z_{\text {den }}^{\prime}$ leads to the same conditions, since the product of all $z_{\text {den }}^{\prime}$ is the same as the product of all $z_{\text {den }}$ that show up in the conformal block. ${ }^{8}$

\section{Restricted instanton partition functions for $\mathcal{M}^{p, p^{\prime}, \mathcal{H}}$. The parameters}

We recall the choice of parameters such that $\mathcal{Z}_{\text {building.block }}^{4 \mathrm{D}}$ reduces to $\mathcal{Z}_{\text {building.block }}^{4 \mathrm{D} \text {, min }}$ which is the building block of Virasoro A-series minimal model conformal blocks times Heisenberg factors.

\subsection{AGT parameterisation of minimal models}

A minimal model $\mathcal{M}^{p, p^{\prime}}$, based on a Virasoro algebra $\mathcal{V}^{p, p^{\prime}}$, characterised by a central charge $c_{p, p^{\prime}}<1$, that we parameterise as

$$
c_{p, p^{\prime}}=1-6\left(a_{p, p^{\prime}}-\frac{1}{a_{p, p^{\prime}}}\right)^{2}, \quad a_{p, p^{\prime}}=\left(\frac{p^{\prime}}{p}\right)^{\frac{1}{2}}
$$

where $\left\{p, p^{\prime}\right\}$ are co-prime integers that satisfy $0<p<p^{\prime}$. In the Coulomb gas approach to computing conformal blocks in minimal models with $c_{p, p^{\prime}}<1[25,26]$, the screening charges $\left\{\alpha_{+}, \alpha_{-}\right\}$, and the background charge $\alpha_{\text {back.ground }}$, satisfy

$$
\alpha_{+}=a_{p, p^{\prime}}, \quad \alpha_{-}=-\frac{1}{a_{p, p^{\prime}}}, \quad \alpha_{\text {back.ground }}=-2 \alpha_{0}, \quad \alpha_{0}=\frac{1}{2}\left(\alpha_{+}+\alpha_{-}\right)
$$

The AGT parameterisation of $\mathcal{M}^{p, p^{\prime}, \mathcal{H}}$ is obtained by choosing

$$
\epsilon_{1}<0<\epsilon_{2}, \quad \epsilon_{1}=\alpha_{-}, \quad \epsilon_{2}=\alpha_{+}
$$

so that $\alpha_{-}<0<\alpha_{+}$. Since we focus on $\mathcal{M}^{p, p^{\prime}, \mathcal{H}}$, we work in terms of $\left\{\alpha_{-}, \alpha_{+}\right\}$instead of $\left\{\epsilon_{1}, \epsilon_{2}\right\}$.

\footnotetext{
${ }^{8}$ In section 10, we outline the derivation of the Burge conditions from $z_{\text {den }}^{\prime}$.
} 


\subsection{Two sets of charges in minimal models}

We consider two types of charges that, in Coulomb gas terms, are expressed in terms of the screening charges $\left\{\alpha_{+}, \alpha_{-}\right\}$. 1. The charge $a_{r, s}$ of the highest weight $\left|a_{r, s}\right\rangle$ of the irreducible highest weight representation $\mathcal{H}_{r, s}^{p, p^{\prime}}$, and 2. The charge $\mu_{r, s}$ of the vertex operator $\mathcal{O}_{\mu}$ that intertwines two highest weight ireducible representations $\mathcal{H}_{r_{1}, s_{1}}^{p, p^{\prime}}$ and $\mathcal{H}_{r_{2}, s_{2}}^{p, p^{\prime}}$. These charges are parameterised in terms of $\alpha_{+}$and $\alpha_{-}$as follows

$$
a_{r, s}=-\frac{r}{2} \alpha_{+}-\frac{s}{2} \alpha_{-}, \quad \mu_{r, s}=-\frac{r}{2} \alpha_{+}-\frac{s}{2} \alpha_{-}+\alpha_{0}, \quad 1 \leqslant r \leqslant p-1,1 \leqslant s \leqslant p^{\prime}-1
$$

\section{From gauge theory parameters to minimal model parameters}

We compare the parameters of $\mathcal{W}^{4 \mathrm{D}}$ and the parameters of $\mathcal{Z}_{\text {building.block }}^{4 \mathrm{D}}$.

We set

$$
\begin{gathered}
a=-\left(\frac{r_{a}}{2}\right) \alpha_{+}-\left(\frac{s_{a}}{2}\right) \alpha_{-}, \quad r_{a} \in\{1,2, \cdots, p-1\}, \quad s_{a} \in\left\{1,2, \cdots, p^{\prime}-1\right\} \\
b=-\left(\frac{r_{b}}{2}\right) \alpha_{+}-\left(\frac{s_{b}}{2}\right) \alpha_{-}, \quad r_{b} \in\{1,2, \cdots, p-1\}, \quad s_{b} \in\left\{1,2, \cdots, p^{\prime}-1\right\} \\
\mu=-\left(\frac{r_{\mu}}{2}\right) \alpha_{+}-\left(\frac{s_{\mu}}{2}\right) \alpha_{-}+\alpha_{0}, \quad r_{\mu} \in\{1,2, \cdots, p-1\}, \quad s_{\mu} \in\left\{1,2, \cdots, p^{\prime}-1\right\}
\end{gathered}
$$

\subsection{The fusion rules}

For completeness, let us mention the fusion rules. In the notation

$$
m_{i}=r_{i}-1, \quad n_{i}=s_{i}-1
$$

the fusion rules take the simple form

$$
m_{a}+m_{b}+m_{\mu}=0 \bmod 2, \quad n_{a}+n_{b}+n_{\mu}=0 \bmod 2,
$$

where the triple $\left\{m_{a}, m_{b}, m_{\mu}\right\}$ satisfies the triangular conditions

$$
m_{a}+m_{b} \geqslant m_{\mu}, \quad m_{b}+m_{\mu} \geqslant m_{a}, \quad m_{\mu}+m_{a} \geqslant m_{b}
$$

with analogous conditions for the triple $\left\{n_{a}, n_{b}, n_{\mu}\right\}$.

\section{Restricted instanton partition functions for $\mathcal{M}^{p, p^{\prime}, \mathcal{H}}$. The partition pairs}

We write the denominator $w_{\operatorname{den}}\left[\mathbf{V}, \mathbf{W}, \boldsymbol{\Delta}, \epsilon_{1}, \epsilon_{2}, g \rightarrow 0\right]$ as $w_{\operatorname{den}}\left[\mathbf{V}, \mathbf{W}, \boldsymbol{\Delta}, \alpha_{+}, \alpha_{-}\right]$, and, following [17], we check the conditions required so that that it has no zeros.

Using $\alpha_{+}$and $\alpha_{-}$, let us write $w_{\text {den }}\left[\mathbf{V}, \mathbf{W}, \boldsymbol{\Delta}, \epsilon_{1}, \epsilon_{2}, R \rightarrow 0\right]$ as $w_{\text {den }}\left[\mathbf{V}, \mathbf{W}, \boldsymbol{\Delta}, \alpha_{+}, \alpha_{-}\right]$, that is

$$
\begin{aligned}
w_{\mathrm{den}}\left[\mathbf{V}, \mathbf{W}, \boldsymbol{\Delta}, \alpha_{+}, \alpha_{-}\right]= & \prod_{\square \in V^{1}}\left(A_{\square, V^{1}}^{++} \alpha_{+}-L_{\square, V^{1}} \alpha_{-}\right) \prod_{\square \in V^{2}}\left(A_{\square, V^{2}}^{++} \alpha_{+}-L_{\square, V^{2}} \alpha_{-}\right) \\
& \times \prod_{\square \in W^{1}}\left(A_{\square, W^{1}} \alpha_{+}-L_{\square, W^{1}}^{++} \alpha_{-}\right) \prod_{\square \in W^{2}}\left(A_{\square, W^{2}} \alpha_{+}-L_{\square, W^{2}}^{++} \alpha_{-}\right)
\end{aligned}
$$




$$
\begin{aligned}
& \times \prod_{\square \in V^{1}}\left(r_{a} \alpha_{+}+s_{a} \alpha_{-}+A_{\square, V^{2}}^{++} \alpha_{+}-L_{\square, V^{1}} \alpha_{-}\right) \\
& \times \prod_{\square \in V^{2}}\left(r_{a} \alpha_{+}+s_{a} \alpha_{-}-A_{\square, V^{1}} \alpha_{+}+L_{\square, V^{2}}^{++} \alpha_{-}\right) \\
& \times \prod_{\square \in W^{1}}\left(r_{b} \alpha_{+}+s_{b} \alpha_{-}+A_{\square, W^{2}} \alpha_{+}-L_{\square, W^{1}}^{++} \alpha_{-}\right) \\
& \times \prod_{\square \in W^{2}}\left(r_{b} \alpha_{+}+s_{b} \alpha_{-}-A_{\square, W^{1}}^{++} \alpha_{+}+L_{\square}, W^{2} \alpha_{-}\right)
\end{aligned}
$$

where we have used

$$
\begin{aligned}
& -2 a=r_{a} \alpha_{+}+s_{a} \alpha_{-}, \quad r=1,2, \cdots \\
& -2 b=r_{b} \alpha_{+}+s_{b} \alpha_{-}, \quad s=1,2, \cdots
\end{aligned}
$$

Consider the denominator $w_{\text {den }}\left[\mathbf{V}, \mathbf{W}, \boldsymbol{\Delta}, \alpha_{+}, \alpha_{-}\right]$in eq. (10.1), on a product by product basis. We need to check the conditions under which any of these products has a zero, then find the restriction that are necessary and sufficient to remove these zeros. The reasoning that we use to obtain these conditions is the same as that in [17]. There are eight products to consider.

\subsection{The initial four products}

In each of the initial four products, the product is over the cells inside a single diagram, thus the arm length $A$ and the leg length $L$ in each of these factors is non-negative. Since $\alpha_{-}<0<\alpha_{+}$, and there is a term $\alpha_{0}>0$ in each factor, the minimal value of each of these factors is greater than zero. Thus there can be no zeros from these factors. To consider the remaining four factors, we require some preparation.

\subsection{Two zero-conditions}

Following [17], we note that, since $\alpha_{-}<0<\alpha_{+}$, any factor of the type that appears in eq. (10.1) has a zero when an equation of type

$$
C_{+} \alpha_{+}+C_{-} \alpha_{-}=0
$$

where $C_{+}, C_{-} \in \mathbb{Z}$, is satisfied. Since $p$ and $p^{\prime}$ are coprime, $\alpha_{-}$and $\alpha_{+}$are $\notin \mathbb{Q}$, the condition in eq. (10.4) is equivalent to the two conditions

$$
C_{+}=c p, \quad C_{-}=c p^{\prime}
$$

are satisfied, where $c$ is a proportionality constant that needs to be determined.

\subsection{From two zero-conditions to one zero-condition}

Consider the two conditions

$$
-A_{\square, i}=A^{\prime} \geqslant 0, \quad L_{\square, j}=L^{\prime} \geqslant 0
$$

which are satisfied if $i \neq j, \square \notin Y^{i}$, and $\square \in Y^{j}$. If $\square$ is in row-R and column-c in $Y^{j}$, then the second condition in (10.6) implies that there is a cell $\boxplus \in Y^{1}$, strictly below $\square$, with 
coordinates $\left\{\mathrm{R}+L^{\prime}, \mathrm{C}\right\}$, such that there are no cells strictly below $\boxplus$. Since there may, or may not, be cells to the right of $\boxplus$, row- $\left(\mathrm{R}+L^{\prime}\right)$ in $Y^{j}$ has length at least $\mathrm{c}$,

$$
y_{\mathrm{R}+L^{\prime}}^{j} \geqslant \mathrm{C}
$$

From the definition of $A_{\square, i}$, we write the first condition in (10.6) as $-A_{\square, i}=A^{\prime}=\mathrm{c}-y_{\mathrm{R}}^{i}$, that is, $\mathrm{c}=A^{\prime}+y_{\mathrm{R}}^{i}$, and using (10.7), we obtain $y_{\mathrm{R}+L^{\prime}}^{j} \geqslant A^{\prime}+y_{\mathrm{R}}^{i}$, which we choose to write as

$$
y_{\mathrm{R}+L^{\prime}}^{j}-y_{\mathrm{R}}^{i} \geqslant A^{\prime}
$$

The condition in eq. (10.8) is equivalent to the two conditions in eq. (10.6).

\subsection{One non-zero condition}

Consider a function $f_{Y^{i}, Y^{j}}$, of a pair of Young diagrams $Y^{i}$ and $Y^{j}, i \neq j$, such that $f_{Y^{i}, Y^{j}}=0$, if and only if $(10.8)$ is satisfied. This implies that $f_{Y^{i}, Y^{j}} \neq 0$, if and only if $Y^{i}$ and $Y^{j}$ satisfies the complementary condition

$$
y_{\mathrm{R}+L^{\prime}}^{j}-y_{\mathrm{R}}^{i}<A^{\prime}
$$

which we choose to write as

$$
y_{\mathrm{R}}^{i}-y_{\mathrm{R}+L^{\prime}}^{j} \geqslant 1-A^{\prime}
$$

\subsubsection{Remark}

In the sequel, we refer to eq. (10.6) as 'zero-conditions', and to eq. (10.10) as 'a non-zerocondition'.

Next we consider the latter four products on the right hand side of eq. (10.1).

\subsection{The first product}

$$
\prod_{\square \in V^{1}}\left(r_{a} \alpha_{+}+s_{a} \alpha_{-}+A_{\square, V^{2}}^{++} \alpha_{+}-L_{\square, V^{1}} \alpha_{-}\right)
$$

vanishes if any factor satisfies

$$
\left(r_{a}+A_{\square, V^{2}}^{++}\right) \alpha_{+}+\left(s_{a}-L_{\square, V^{1}}\right) \alpha_{-}=0
$$

which leads to the conditions

$$
-A_{\square, V^{2}}=r_{a}+1+c p, \quad L_{\square, V^{1}}=s_{a}+c p^{\prime}
$$

Since $\square \in V^{1}, L_{\square, V^{1}} \geqslant 0$. Given that $s$ and $p^{\prime}$ are non-zero positive integers, the second equation in eq. (10.13) admits a solution only if $c=0,1, \cdots$ The first equation in eq. (10.13) admits a solution if $\square \notin V^{2}$.

\subsubsection{From two zero-conditions to one non-zero-condition}

Following paragraphs 10.3 and 10.4, the two zero-conditions in (10.13) are equivalent to one non-zero-condition,

$$
V_{\mathrm{R}}^{2}-V_{\mathrm{R}+s+c p^{\prime}}^{1} \geqslant-r-c p
$$




\subsubsection{The stronger condition}

Eq. (10.14) is the statement that to eliminate the zeros, we want $V_{\mathrm{R}}^{2}-V_{\mathrm{R}+s+c p^{\prime}}^{1} \geqslant-r-$ $c p$, where $c=\{0,1, \cdots\}$ Since the row-lengths of a partition are by definition weakly decreasing, and $c=\{0,1, \cdots\}$, this is the case if $V_{\mathrm{R}}^{2}-V_{\mathrm{R}+s}^{1} \geqslant-r-c p$, which is the case if $V_{\mathrm{R}}^{2}-V_{\mathrm{R}+s}^{1} \geqslant-r$. Thus, we should set $c=0$, and obtain

$$
V_{\mathrm{R}}^{2}-V_{\mathrm{R}+s}^{1} \geqslant-r
$$

\subsection{The second product}

$$
\prod_{\square \in V^{2}}\left(r_{a} \alpha_{+}+s_{a} \alpha_{-}-A_{\square, V^{1}} \alpha_{+}+L_{\square, V^{2}}^{++} \alpha_{-}\right)
$$

vanishes if any factor satisfies

$$
\left(r_{a}-A_{\square, V^{1}}\right) \alpha_{+}+\left(s_{a}+L_{\square, V^{2}}^{++}\right) \alpha_{-}=0
$$

which leads to the conditions

$$
-A_{\square, V^{1}}=-r_{a}+c p, \quad L_{\square, V^{2}}=-s_{a}-1+c p^{\prime},
$$

Since $\square \in V^{2}, L_{\square, V^{2}} \geqslant 0$. Given that $s_{a}$ and $p^{\prime}$ are non-zero positive integers, the second equation in eq. (10.18) admits a solution only if $c=1, \cdots$ The first equation in eq. (10.18) admits a solution if $\square \notin V^{1}$.

\subsubsection{From two zero-conditions to one non-zero-condition}

Following paragraphs 10.3 and 10.4, the two zero-conditions in eq. (10.18) are equivalent to one non-zero-condition,

$$
V_{\mathrm{R}}^{1}-V_{\mathrm{R}-1-s_{a}+c p^{\prime}}^{2} \geqslant 1+r_{a}-c p
$$

\subsubsection{The stronger condition}

Eq. (10.19) is the statement that to eliminate the zeros, we want $V_{\mathrm{R}}^{1}-V_{\mathrm{R}-1-s_{a}+c p^{\prime}}^{2} \geqslant$ $1+r_{a}-c p$, where $c=\{1,2, \cdots\}$. Since the row-lengths of a partition are by definition weakly decreasing, and $c=\{1,2, \cdots\}$, this is the case if $V_{\mathrm{R}}^{1}-V^{2, \mathrm{R}+p^{\prime}-s_{a}-1} \geqslant 1+r_{a}-c p$. In turn, is the case if $V_{\mathrm{R}}^{1}-V_{\mathrm{R}+p^{\prime}-s_{a}-1}^{2} \geqslant 1+r_{a}-p$. Thus, we should set $c=1$, to obtain

$$
V_{\mathrm{R}}^{1}-V_{\mathrm{R}+\left[p^{\prime}-s_{a}\right]-1}^{2} \geqslant 1-\left(p-r_{a}\right)
$$

\subsection{The third product}

$$
\prod_{\mathbf{\square} \in W^{1}}\left(r_{b} \alpha_{+}+s_{b} \alpha_{-}+A_{\mathbf{m}, W^{2}} \alpha_{+}-L_{\mathbf{m}, W^{1}}^{++} \alpha_{-}\right)
$$

vanishes if any factor satisfies

$$
\left(r_{b}+A_{\mathbf{\square}, W^{2}}\right) \alpha_{+}+\left(s_{b}-L_{\mathbf{\square}, W^{1}}^{++}\right) \alpha_{-}=0
$$

which leads to the conditions

$$
-A_{\square}, W^{2}=r_{b}+c p, \quad L_{\mathbf{\square}, W^{1}}=-1+s_{b}+c p^{\prime},
$$




\subsubsection{The stronger condition}

Using the same arguments as in subsections 10.5 and 10.6, are possible for $c=0,1, \ldots$, $\mathbf{\square} \in W^{1}, \boldsymbol{\square} \notin W^{2}$, and we should choose $c=0$ to obtain

$$
W_{\mathrm{R}}^{2}-W_{\mathrm{R}+s-1}^{1} \geqslant 1-r
$$

\subsection{The fourth product}

$$
\prod_{\mathbf{\square} \in W^{2}}\left(r_{b} \alpha_{+}+s_{b} \alpha_{-}-A_{\mathbf{m}, W^{1}}^{++} \alpha_{+}+L_{\mathbf{m}, W^{2}} \alpha_{-}\right)
$$

vanishes if any factor satisfies

$$
\left(r_{b}-A_{\square, W^{1}}^{++}\right) \alpha_{+}+\left(s_{b}+L_{\mathbf{m}, W^{2}}\right) \alpha_{-}=0,
$$

which leads to the conditions

$$
-A_{\mathbf{\square}, W^{1}}=1-r_{b}+c p, \quad L_{\mathbf{\square}, W^{2}}=-s_{b}+c p^{\prime}
$$

\subsubsection{The stronger condition}

Using the same arguments as in subsections 10.5 and 10.6, are possible for $c=1,2, \cdots$, $\square \in W^{2}$ and $\square \notin W^{1}$, and we should choose $c=0$ to obtain

$$
W_{\mathrm{R}}^{1}-W_{\mathrm{R}+\left[p^{\prime}-s_{b}\right]}^{2} \geqslant-\left(p-r_{b}\right)
$$

\subsection{The Burge conditions}

Eqs. (10.15) and (10.20) are conditions on the partition pair $\mathbf{V}$ on one side of $\mathcal{Z}_{\text {building.block, }}$, while eqs. (10.24) and (10.28) are conditions on the partition pair $\mathbf{W}$ on the other side

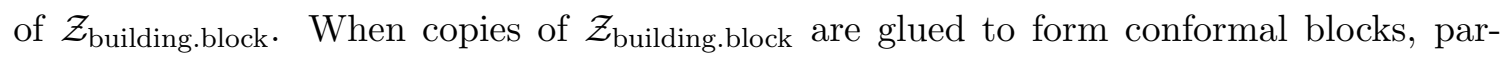
tition pairs on one side are identified with partition pairs on the other side. Thus each partition pair must satisfy all conditions. However, these conditions are not independent as two of them are satisfied when the other two are satisfied. More specifically, following paragraphs 10.3 and 10.4, we can see that it is sufficient to enforce the two conditions in eqs. (10.20) and (10.24),

$$
\mu_{\mathrm{R}}-\mu_{\mathrm{R}+\left[p^{\prime}-s\right]-1} \geqslant 1-(p-r), \quad \nu_{\mathrm{R}}-\nu_{\mathrm{R}+s-1} \geqslant 1-r
$$

which we write in terms of a partition pair $\{\mu, \nu\}$ that could be on either side of

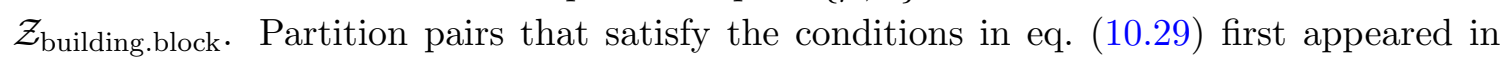
the work of W H Burge on Rogers-Ramanujan-type identities [27]. The appeared in earlier studies of Virasoro characters in [28-30] and more recently in the context of the AGT correspondence in $[16,17]$. 


\section{Comments and open questions}

Outline of result. We can generate conformal blocks of Virasoro $A$-series minimal models, labelled by the co-primes $p$ and $p^{\prime}$, times a Heisenberg factor, as follows. 1. Start from the refined topological vertex of [9] defined in eq. (2.10), 2. Glue four copies of the refined topological vertex to produce a $5 \mathrm{D} \mathrm{U}(2)$ basic web partition function, then take the $R \rightarrow 0$ limit, to obtain its $4 \mathrm{D}$ counterpart $\mathcal{W}_{\mathbf{V W} \boldsymbol{\Delta}}^{4 \mathrm{D}}$ as in eqs. (3.17), (3.18), (3.19). 3. Set the Kähler parameters $\boldsymbol{\Delta}$, and the deformation parameters $q$ and $t$ in $\mathcal{W}_{\mathbf{V W} \boldsymbol{\Delta}}^{4 \mathrm{D}}$ as in eqs. (6.2), (8.2), (8.3), and (8.4), and 4 . require each of the partition pairs $\mathbf{V}$ and $\mathbf{W}$ to satisfy the Burge conditions in eq. (10.29).

The refined topological vertex of Awata and Kanno. We have used the refined topological vertex of Iqbal, Kozcaz and Vafa [9], but could have equally well used that of Awata and Kanno [7, 8]. The two vertices are equivalent as explained in [31].

Layers. The topic discussed in this note is vast and consists of many layers. We could have started our discussion from M-theory and used the language of M5 branes, but we decided to stay away from this, in this short note. Instead, we started from A-model topological strings, which live in a corner of the M-theory. From the refined topological vertex and topological strings, we obtained the building block of the instanton partition function of a 5D quiver gauge theory. We could have used the K-theoretic version of the AGT correspondence to obtain the minimal model analogues of the $q$-deformed Liouville conformal blocks discussed in [32-37]. Instead, we skipped the $q$-deformed blocks, took the $4 \mathrm{D}$ limit, and used the 4D version of AGT to obtain minimal model conformal blocks. The M-theoretic origins of the minimal conformal blocks and their $q$-deformations should be topics of separate studies.

Interpretation. Missing from this note is an interpretation of the minimal model parameters in topological string or gauge theory terms. A complete interpretation will require working in M-theory terms which lies outside the scope of this work. In particular, missing is an interpretation of the Burge conditions in topological string or gauge theory terms. We conjecture that such interpretations require re-derivations of existing results in ways that allow ab initio for minimal model parameters. Current derivations do not do that, and for that reason, one obtains results that are not well-defined upon substitution of minimal-model parameters and that require a posteriori restrictions as in $[16,17] .{ }^{9}$ We plan to address this topic in future work.

Postscript. Following the completion of work on this note, we became aware of the fact that $\mathrm{U}(N)$ versions of eqs. 2.14 and $\mathbf{2 . 1 7}$ in this note, were obtained in eq. 4.67 in [22], and in eq. $\mathbf{5 . 1}$ and subsequent equations in [38]. ${ }^{10}$

\footnotetext{
${ }^{9}$ OF wishes to thank J-E Bourgine for discussions on this point.

${ }^{10}$ The basic web in this note is related, by 'a flop' to the a strip in [22]. This term was introduced in [39], where strip partition functions were studied in the context of the original, unrefined topological vertex.
} 


\section{Acknowledgments}

OF would like to thank M Bershtein for collaboration on [17], results of which were used in this note, and H Awata, J-E Bourgine, D Krefl, V Mitev, E Pomoni, A Tanzini and Y Zenkevich for discussions and useful remarks at various stages of work on this note. We thank the anonymous referee for useful comments that helped us improve the presentationh. OF is supported by the Australian Research Council [ARC].

Open Access. This article is distributed under the terms of the Creative Commons Attribution License (CC-BY 4.0), which permits any use, distribution and reproduction in any medium, provided the original author(s) and source are credited.

\section{References}

[1] A. Neitzke and C. Vafa, Topological strings and their physical applications, hep-th/0410178 [INSPIRE].

[2] M. Mariño, Chern-Simons theory and topological strings, Rev. Mod. Phys. 77 (2005) 675 [hep-th/0406005] [INSPIRE].

[3] M Mariño, Chern-Simons theory, matrix models, and topological strings, Int. Ser. Monogr. Phys. 131 (2005) 1, Oxford U.K. (2005) [INSPIRE].

[4] S.H. Katz, A. Klemm and C. Vafa, Geometric engineering of quantum field theories, Nucl. Phys. B 497 (1997) 173 [hep-th/9609239] [INSPIRE].

[5] S. Katz, P. Mayr and C. Vafa, Mirror symmetry and exact solution of $4 D N=2$ gauge theories: 1, Adv. Theor. Math. Phys. 1 (1998) 53 [hep-th/9706110] [INSPIRE].

[6] M. Aganagic, A. Klemm, M. Mariño and C. Vafa, The topological vertex, Commun. Math. Phys. 254 (2005) 425 [hep-th/0305132] [INSPIRE].

[7] H. Awata and H. Kanno, Instanton counting, Macdonald functions and the moduli space of D-branes, JHEP 05 (2005) 039 [hep-th/0502061] [INSPIRE].

[8] H. Awata and H. Kanno, Refined BPS state counting from Nekrasov's formula and Macdonald functions, Int. J. Mod. Phys. A 24 (2009) 2253 [arXiv:0805.0191] [InSPIRE].

[9] A. Iqbal, C. Kozcaz and C. Vafa, The refined topological vertex, JHEP 10 (2009) 069 [hep-th/0701156] [INSPIRE].

[10] N.A. Nekrasov, Seiberg-Witten prepotential from instanton counting, Adv. Theor. Math. Phys. 7 (2004) 831 [hep-th/0206161] [INSPIRE].

[11] T.J. Hollowood, A. Iqbal and C. Vafa, Matrix models, geometric engineering and elliptic genera, JHEP 03 (2008) 069 [hep-th/0310272] [INSPIRE].

[12] T. Eguchi and H. Kanno, Topological strings and Nekrasov's formulas, JHEP 12 (2003) 006 [hep-th/0310235] [INSPIRE].

[13] J. Zhou, Curve counting and instanton counting, math/0311237 [INSPIRE].

[14] M. Taki, Refined topological vertex and instanton counting, JHEP 03 (2008) 048 [arXiv:0710.1776] [INSPIRE]. 
[15] L.F. Alday, D. Gaiotto and Y. Tachikawa, Liouville correlation functions from four-dimensional gauge theories, Lett. Math. Phys. 91 (2010) 167 [arXiv:0906.3219] [INSPIRE].

[16] K.B. Alkalaev and V.A. Belavin, Conformal blocks of $W_{N}$ minimal models and AGT correspondence, JHEP 07 (2014) 024 [arXiv: 1404.7094] [INSPIRE].

[17] M. Bershtein and O. Foda, AGT, Burge pairs and minimal models, JHEP 06 (2014) 177 [arXiv: 1404.7075] [INSPIRE].

[18] V.A. Fateev and A.V. Litvinov, Integrable structure, $W$-symmetry and AGT relation, JHEP 01 (2012) 051 [arXiv: 1109.4042] [INSPIRE].

[19] N. Wyllard, $A_{N-1}$ conformal Toda field theory correlation functions from conformal $N=2$ $\mathrm{SU}(N)$ quiver gauge theories, JHEP 11 (2009) 002 [arXiv:0907.2189] [INSPIRE].

[20] V. Mitev and E. Pomoni, Toda 3-point functions from topological strings, JHEP 06 (2015) 049 [arXiv: 1409.6313] [INSPIRE].

[21] M. Isachenkov, V. Mitev and E. Pomoni, Toda 3-point functions from topological strings II, arXiv:1412.3395 [INSPIRE].

[22] L. Bao, V. Mitev, E. Pomoni, M. Taki and F. Yagi, Non-Lagrangian theories from brane junctions, JHEP 01 (2014) 175 [arXiv:1310.3841] [INSPIRE].

[23] I.G. Macdonald, Symmetric functions and Hall polynomials, $2^{\text {nd }}$ edition, Clarendon Press, Oxford U.K. (1995).

[24] H. Nakajima and K. Yoshioka, Instanton counting on blowup. 1, Invent. Math. 162 (2005) 313 [math/0306198] [INSPIRE].

[25] B. Nienhuis, Coulomb gas representations of phase transitions in two dimensions, Phase Transitions and Critical Phenomena 11 (1987) 1.

[26] V.S. Dotsenko and V.A. Fateev, Conformal algebra and multipoint correlation functions in two-dimensional statistical models, Nucl. Phys. B 240 (1984) 312 [INSPIRE].

[27] W.H. Burge, Restricted partition pairs, J. Combinat. Theor. A 63 (1993) 210.

[28] O. Foda, K.S.M. Lee and T.A. Welsh, A Burge tree of Virasoro type polynomial identities, Int. J. Mod. Phys. A 13 (1998) 4967 [q-alg/9710025] [INSPIRE].

[29] O. Foda and T.A. Welsh, On the combinatorics of Forrester-Baxter models, in Physical Combinatorics, M. Kashiwara and T. Miwa eds., Progr. Math. 191 (2000) 49, Birkhauser, Boston U.S.A. (2000) [math.QA/0002100] [INSPIRE].

[30] T.A. Welsh, Fermionic expressions for minimal model Virasoro characters, Mem. Am. Math. Soc. 175N827 (2005) 1 [math/0212154] [INSPIRE].

[31] H. Awata, B. Feigin and J. Shiraishi, Quantum algebraic approach to refined topological vertex, JHEP 03 (2012) 041 [arXiv:1112.6074] [INSPIRE].

[32] H. Awata and Y. Yamada, Five-dimensional AGT conjecture and the deformed Virasoro algebra, JHEP 01 (2010) 125 [arXiv:0910.4431] [INSPIRE].

[33] H. Awata and Y. Yamada, Five-dimensional AGT relation and the deformed $\beta$-ensemble, Prog. Theor. Phys. 124 (2010) 227 [arXiv:1004.5122] [INSPIRE].

[34] M. Aganagic, N. Haouzi, C. Kozcaz and S. Shakirov, Gauge/Liouville triality, arXiv:1309.1687 [INSPIRE]. 
[35] M. Aganagic, N. Haouzi and S. Shakirov, $A_{n}$-triality, arXiv:1403.3657 [INSPIRE].

[36] F. Nieri, S. Pasquetti and F. Passerini, $3 D$ and $5 D$ gauge theory partition functions as q-deformed CFT correlators, Lett. Math. Phys. 105 (2015) 109 [arXiv:1303.2626] [INSPIRE].

[37] F. Nieri, S. Pasquetti, F. Passerini and A. Torrielli, 5D partition functions, $q$-Virasoro systems and integrable spin-chains, JHEP 12 (2014) 040 [arXiv:1312.1294] [INSPIRE].

[38] V. Mitev, E. Pomoni, M. Taki and F. Yagi, Fiber-base duality and global symmetry enhancement, JHEP 04 (2015) 052 [arXiv: 1411.2450] [INSPIRE].

[39] A. Iqbal and A.-K. Kashani-Poor, The vertex on a strip, Adv. Theor. Math. Phys. 10 (2006) 317 [hep-th/0410174] [INSPIRE]. 